\title{
Scatter modelling and compensation in emission tomography
}

\author{
Habib Zaidi ${ }^{1}$, Kenneth F. Koral ${ }^{2}$ \\ 1 Division of Nuclear Medicine, Geneva University Hospital, Geneva, Switzerland \\ 2 Department of Radiology, University of Michigan Medical Center, Ann Arbor, Michigan, USA \\ Published online: 31 March 2004 \\ (C) Springer-Verlag 2004
}

\begin{abstract}
In nuclear medicine, clinical assessment and diagnosis are generally based on qualitative assessment of the distribution pattern of radiotracers used. In addition, emission tomography (SPECT and PET) imaging methods offer the possibility of quantitative assessment of tracer concentration in vivo to quantify relevant parameters in clinical and research settings, provided accurate correction for the physical degrading factors (e.g. attenuation, scatter, partial volume effects) hampering their quantitative accuracy are applied. This review addresses the problem of Compton scattering as the dominant photon interaction phenomenon in emission tomography and discusses its impact on both the quality of reconstructed clinical images and the accuracy of quantitative analysis. After a general introduction, there is a section in which scatter modelling in uniform and non-uniform media is described in detail. This is followed by an overview of scatter compensation techniques and evaluation strategies used for the assessment of these correction methods. In the process, emphasis is placed on the clinical impact of image degradation due to Compton scattering. This, in turn, stresses the need for implementation of more accurate algorithms in software supplied by scanner manufacturers, although the choice of a general-purpose algorithm or algorithms may be difficult.
\end{abstract}

Keywords: Emission tomography - Scatter modelling Scatter correction - Reconstruction - Quantification

Eur J Nucl Med Mol Imaging (2004) 31:761-782

DOI $10.1007 / \mathrm{s} 00259-004-1495-\mathrm{Z}$

Habib Zaidi (

Division of Nuclear Medicine, Geneva University Hospital, 1211 Geneva, Switzerland e-mail: habib.zaidi@hcuge.ch

Tel.: +41-22-3727258, Fax: +41-22-3727169

\section{Introduction}

In order to discuss photon scattering, one must first define it. At this point, it is important to distinguish between coherent (Rayleigh) and incoherent (Compton) scattering. Coherent scattering of a photon involves an interaction with an atom so there is virtually no loss of energy. In addition, it usually involves only a small change in direction for the incoming photon. For these reasons, coherently scattered photons can be included with the primaries - that is, there is usually no reason to eliminate them or to correct for their existence. Moreover, their occurrence is much less likely than the occurrence of Compton-scattered photons for the radionuclides frequently used in nuclear medicine. Therefore, we will say nothing further about coherently scattered photons, and the term "scattering" from here on will mean Compton scattering. Before briefly discussing the characteristics of Compton scattering, it is also useful to say that for the purposes of this review, a Compton scatter event's location will usually either be in the patient or in the collimator septa of the imaging detection system. In stipulating this requirement, we are neglecting the possibility of scatter in the gantry or table that supports the patient. In practice, such scattering exists, but it is probably of small magnitude. The problem is that in physical measurements it is usually present, although often not mentioned, whereas in simulations it is often not included. One could therefore say that we are describing the typical simulation study.

In single-photon emission tomography (SPECT), for radionuclides which have a photon emission above the photopeak window of interest, backscatter of a highenergy photon from behind the crystal back into it can lead to extra scatter counts. However, in the case of a ${ }^{111}$ In point source in air, for example, $247-\mathrm{keV}$ photons that backscatter, are detected and yield a signal which falls within the photopeak window of the $172-\mathrm{keV}$ emission contribute "at most a few percent of the total counts" within that window [1]. Therefore, even here it appears they can be neglected. In cases where scattering from such locations is more important or has been included, we will explicitly point out the fact. We also as- 
Fig. 1. A Schematic diagram of the origin and shape of detector scatter component for a cylindrical multi-ring PET scanner geometry estimated from a measurement in air using a line source. B Schematic diagram of the origin and shape of object scatter component estimated from measurements in a cylindrical phantom using a centred line source. Both single and multiple scatter are illustrated

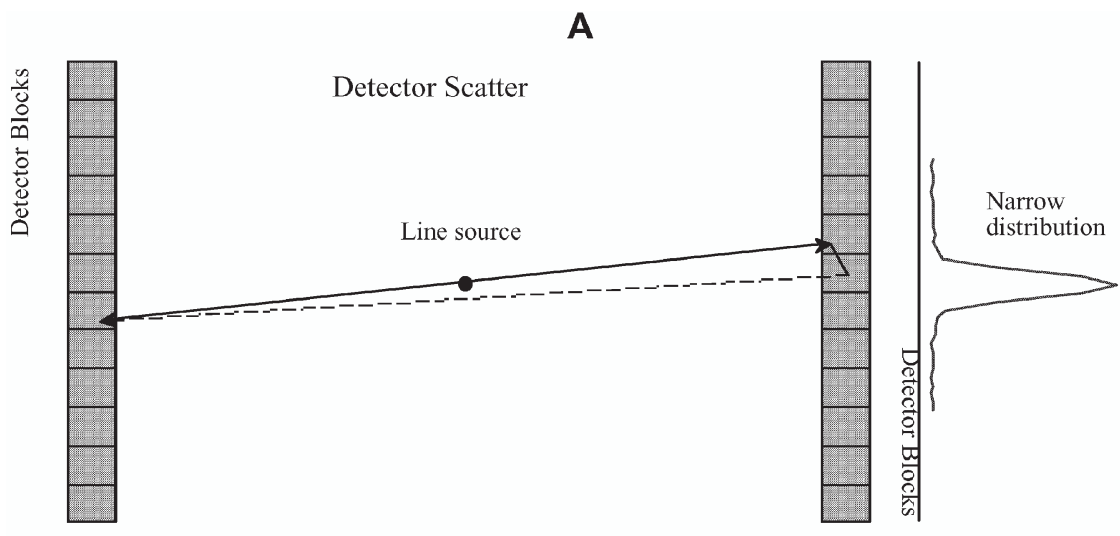

B

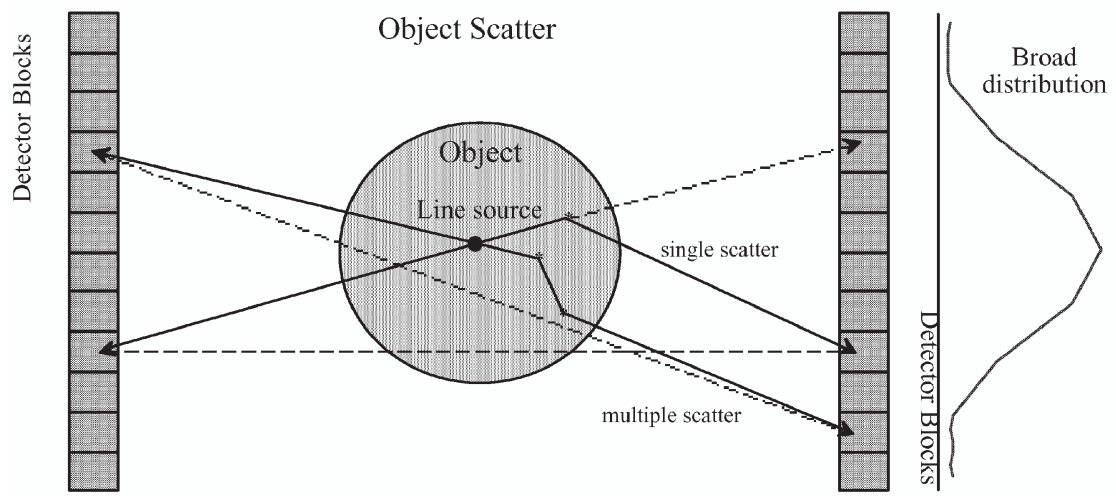

sume that detection can be correctly characterised, and we will not discuss steps in that process. That is, we are declining to distinguish between detection by photoelectric absorption and that based upon at least one Compton scatter. Some authors have gone into that process while covering scatter and its correction $[2,3,4,5]$, but we take the point of view that discussing it here would only confuse matters. We feel this point of view is supported by the fact that scatter correction usually does not involve correcting for scatter in the detector crystal. Figure 1 illustrates the difference in terms of origin and shape between object and detector scatter components.

In Compton scattering, the scattered photon emerges from the interaction point with less energy than it had originally, and with a change of direction. The usual physics textbook algebraic equation for the final energy as a function of the initial energy and of the angular change of direction assumes that the interaction was with a free electron at rest [6]. There are corrections to that equation which take into account the fact that the electron actually is moving and is bound to an atom. The result is that the photons that have scattered through a given angle actually have a distribution of energies sharply peaked about the value calculated by the simple formula [7]. Although this effect, which is called Doppler broadening, has some importance for Compton scatter cameras [8], we will not need to discuss it further here because the energy distribution is so sharply peaked. There are two things about Compton scattering that are important to note for our purposes. One is that the loss of energy can lead to the elimination of a Compton-scattered photon by the lower energy window looking at the detected signal. When this happens, the event is no longer of importance for scatter correction. The other is that the change of direction is the basic cause of the problem that calls for correction. Because of the direction change, the detected scattered photon is tracked back incorrectly during reconstruction, if it is assumed to be from an emission site. There will be more discussion of these matters further into this review.

The formula which gives the probability of a Compton scatter from a free electron through a given angle is the Klein-Nishina formula. This formula can also be found in physics textbooks, in an upcoming book that gives a comprehensive view of quantitative nuclear imaging [9] and in many other places.

We have two final introductory points:

1. A Compton-scattered photon can have Compton scattered multiple times in either the patient or the collimator of the detection system, or a certain number of times (one or more) in the patient, and then a certain number of times (one or more) in the collimator of the detection system. 
Table 1. Magnitude of collimator scatter and penetration for the range of radionuclides employed in SPETa

\begin{tabular}{|c|c|c|c|c|c|}
\hline \multirow[t]{2}{*}{ Source geometry } & \multirow{2}{*}{$\begin{array}{l}\text { Radionuclide } \\
\text { (window) }\end{array}$} & \multirow[t]{2}{*}{ Collimator type } & \multicolumn{3}{|c|}{ Photon's method of reaching detector } \\
\hline & & & $\begin{array}{l}\text { Passed along } \\
\text { collimator } \\
\text { channel }\end{array}$ & $\begin{array}{l}\text { Penetrated } \\
\text { one or more } \\
\text { collimator } \\
\text { septa }\end{array}$ & $\begin{array}{l}\text { Scattered at } \\
\text { least once in } \\
\text { collimator } \\
\text { septa }\end{array}$ \\
\hline Small source in air & ${ }^{67} \mathrm{Ga}^{\mathrm{b}}(93-\mathrm{keV}$ peak) & ME & $91.1 \%$ & $3.4 \%$ & $2.6 \%$ \\
\hline Point source in air & $99 \mathrm{mTc}^{\mathrm{c}}(141 \mathrm{keV})$ & $\mathrm{LE}$ & $94.5 \%$ & $3.6 \%$ & $1.9 \%$ \\
\hline \multirow[t]{2}{*}{ Point source in cold cylinder } & ${ }^{111} \operatorname{In}^{\mathrm{d}}(172-\mathrm{keV}$ peak) & \multirow[t]{2}{*}{ Optimal $\left(\right.$ lead content $\left.=14 \mathrm{~g} / \mathrm{cm}^{2}\right)$} & $89.3 \%$ & $7.3 \%$ & $3.4 \%$ \\
\hline & ${ }^{111} \mathrm{In}^{\mathrm{d}}(247-\mathrm{keV}$ peak) & & $49.9 \%$ & $34.6 \%$ & $15.5 \%$ \\
\hline Small source in air & ${ }^{67} \mathrm{Ga}(300-\mathrm{keV}$ peak) & ME & $45.6 \%$ & $32.3 \%$ & $22.1 \%$ \\
\hline \multirow[t]{2}{*}{ Point source in air } & ${ }^{131}$ I (360 keV peak) & $\mathrm{HE}$ & $27.3 \%$ & $43.3 \%$ & $29.4 \%$ \\
\hline & & UHE & $72.3 \%$ & $17.3 \%$ & $10.3 \%$ \\
\hline
\end{tabular}

LE, Low energy; ME, medium energy; HE, high energy; UHE, ultra high energy

${ }^{a}$ For all detected photons with an energy signal within the photopeak window indicated, the percentage associated with a given path from source to detector is indicated. Counts over the entire projection image were included

b Values do not add up to $100 \%$ because for this window there was a $2.9 \%$ contribution to all counts specifically from $\mathrm{Pb} \mathrm{X}$-rays produced in the collimator

2. The emission site of the original photon may be outside of the field of view of the scanner.

Sometimes, both of these possibilities are neglected in formulating the correction. In those cases, they can be investigated as additional effects which led to bias in the corrected estimate. Here bias is a statistical term that is defined as a displacement, either up or down, in the strength of a voxel in the resulting image compared with the true (or ideal) value.

\section{Magnitude of scatter}

A general idea of the magnitude of collimator scatter and penetration for the range of radionuclides employed in SPECT is given in Table 1. Patient scatter is not involved for ${ }^{99 \mathrm{~m} T c}$ [10], ${ }^{67} \mathrm{Ga}$ [11] or ${ }^{131} \mathrm{I}$ [12], because the source was simulated in air. The values for ${ }^{111} \operatorname{In}$ [13] are appropriate for inclusion in the table, because in that case the definition of a scatter count excluded object scatter (de Vries, personal communication). It can be seen that collimator scatter increases as the energy of the photopeak of interest increases from a low of $1.9 \%$ for ${ }^{99 \mathrm{mTc}}(141 \mathrm{keV})$ to a high of $29.4 \%$ for ${ }^{131} \mathrm{I}(364 \mathrm{keV})$ with the usual high-energy collimator. The penetration percentage also increases with energy. These same tendencies were previously observed in a single study using four different energy emissions from ${ }^{67} \mathrm{Ga}$ [11]. Therefore, correction for photons that penetrate through, or scatter in, collimator septa is hardly important at all for c The window was actually set for the $159-\mathrm{keV}$ emission of ${ }^{123}$ I [10]. Thus it is offset high for the only emission from ${ }^{99 \mathrm{mTc}}(141 \mathrm{keV})$ d In this case, by definition scatter events only included scatter in the collimator, which could be Rayleigh or Compton scattering (the value is thus lower than it otherwise would be). Therefore, also, the column for "Passed along collimator channel" includes both the counts implied by the label and the counts from photons that scattered in the cylinder (the value is thus higher than it otherwise would be) [13] (de Vries, personal communication)

${ }^{99 \mathrm{~m} T c}$, but is potentially important for radionuclides with higher-energy emissions. Note that an ultra-high-energy collimator with twice the septal thickness can decrease both the collimator scatter and the penetration, as shown by the values for ${ }^{131} \mathrm{I}$ in Table 1 . Unfortunately, a similar table is available neither for a point source in a scattering medium nor for an object containing a distribution of activity. It is, therefore, possible that the dependence on energy could be different in these cases.

A general idea of the magnitude of scatter in myocardial imaging is an estimate that the ratio of scattered to unscattered (primary) counts, $S P$, is approximately 0.34 for ${ }^{99 \mathrm{~m}} \mathrm{Tc}$ and 0.95 for ${ }^{201} \mathrm{Tl}$ [14]. The magnitude of different types of events for a ${ }^{131}$ I source surrounded by a scattering medium is given in Table 2 (Dewaraja, personal communication). With the standard high-energy collimator, $43 \%$ of all detected counts are scattered in either the object or the collimator or both. Also, $27 \%$ of all detected counts solely penetrate one or more collimator septa. It has been shown for the region of interest and phantom of Table 2 that the spectrum from such penetrating 364-keV photons is the same as that from the photons that pass along a collimator channel [12] and so one cannot discriminate between the two. One can conjecture that multi-window scatter correction methods cannot distinguish between the two in general. Therefore, scatter correction for ${ }^{131}$ I generally does not include correction for penetration of $364-\mathrm{keV}$ gammas. For the case above, then, $57 \%$ of counts $(30 \%$ passing along a collimator channel plus $27 \%$ penetrating one or more septa) are considered "good" counts. That still leaves a 
Table 2. Magnitude of different types of events for a ${ }^{131}$ I source surrounded by a scattering medium ${ }^{\mathrm{a}}$

\begin{tabular}{llll}
\hline Collimator type & \multicolumn{2}{l}{ Photon's method of reaching detector } & \\
\cline { 2 - 4 } & $\begin{array}{l}\text { Passed along collimator } \\
\text { channel }\end{array}$ & $\begin{array}{l}\text { Penetrated one or more } \\
\text { collimator septa }\end{array}$ & $\begin{array}{l}\text { Scattered at least once in object } \\
\text { or in collimator septa }\end{array}$ \\
\hline HE & $30 \%$ & $27 \%$ & $43 \%$ \\
UHE & $61 \%$ & $13 \%$ & $26 \%$ \\
\hline
\end{tabular}

HE, High energy; UHE, ultra high energy

${ }^{a}$ For all detected photons with an energy signal within the $20 \%$ photopeak window of the main $364-\mathrm{keV}$ emission of ${ }^{131} \mathrm{I}$, the percentage of the total associated with a given path from source to detector is indicated. The source was a $7.4-\mathrm{cm}$ diameter hot sphere centrally located in a warm cylinder with a diameter of $22 \mathrm{~cm}$ and a height of $21 \mathrm{~cm}$. Only counts detected in a circle in the projec-

sizeable $43 \%$ needing correction. Note, however, that with the ultra-high-energy collimator, the problem is reduced: $74 \%$ are "good" counts and $26 \%$ need correction.

In positron emission tomography (PET), the magnitude of the included scatter depends heavily on the acquisition mode, the body section being imaged (e.g. brain versus thorax versus abdomen or pelvis), and the placement and width of the energy signal acceptance window. The mode depends on whether the field of view for a given detector is restricted in the axial direction (along the z-axis) by the placement of lead or tungsten septa (2D mode), or left considerably more open (3D mode). For standard acceptance windows, in 2D mode the scatter is " $10-20 \%$ of the total counts acquired" and in 3D mode "approaches half of all recorded events" [15, 16].

It has been shown [17] that in 3D acquisition mode, the variation of the scatter fraction as a function of the phantom size is not linear, reaching a maximum of $66 \%$ for a point source located in the centre of a cylindrical phantom (diameter $50 \mathrm{~cm}$, height $20 \mathrm{~cm}$ ). It is worth emphasising that the scatter fraction for the same point source in air is higher in $2 \mathrm{D}(6 \%)$ than in 3D mode $(2 \%)$ owing to the contribution of scatter in the septa in the former case. Another concern in 3D PET in contrast to 2D PET is the scatter contribution from activity outside the field of view and multiple scatter.

\section{Importance of scatter}

In the earliest literature on scatter correction, the main import of scatter was considered to be a loss of contrast in the image. In the simplest of descriptions, this means that a true zero in a reconstructed image occurs as a positive value. This effect was demonstrated by imaging non-radioactive spheres in a radioactivity surround [18]. The corruption was frequently described as a pedestal upon which the true image sat. It was soon realised that for quantitative imaging, Compton scatter causes a more tion image that corresponded to the sphere were included. N.B. Photons which scattered at least once in the object and also penetrated collimator septa were included in the scattered percentage. Also, photons which had an emission energy higher than $364 \mathrm{keV}$ and backscattered from material behind the scintillation crystal were included in the scattered percentage, but their numbers were small

complicated distortion in at least parts of the image. In cardiology with $99 \mathrm{~m} \mathrm{Tc}$, King et al., using Monte Carlo simulation of a uniformly perfused left ventricle and employing a bull's eye polar map of counts, pointed out that after attenuation correction using true linear attenuation coefficients the total change in counts due to scatter was $31.3 \%$ and that the shape of the distortion was such that there was a slight increase in apparent activity as one moved from the apex of the heart towards the base [14]. So, to the extent that clinicians want an accurate quantitative image, including the best contrast possible, scatter is always a problem. The extent to which it can be shown to have a disabling effect upon the goal for which the image is to be employed is a much more difficult matter to discuss and to document. We will try to point out specific instances in this review.

\section{Relationship of scatter to attenuation}

In all nuclear medicine imaging (single-photon planar, SPECT and PET), patient scatter is the companion of patient attenuation. That is, a large fraction of the photons that are attenuated instantly fall into the category of a potential scatter-corrupting photon. A photoelectric absorption contributes only to attenuation, but a Compton scatter interaction increases attenuation and also sets up a potential scatter corruption. For the potentiality to become a reality, the scattered photon must be detected and also must fall within the energy-signal acceptance window. The sole purpose of that window is to work against the acceptance of scattered photons. An important difference between Compton scatter in SPECT and PET is that in the former, scatter events carry information that can be useful for determination of the body outline or of the non-uniform attenuation map. For example, Pan et al. estimated the regions of the lungs and non-pulmonary tissues of the chest by segmenting the photopeak and Compton scatter window images to estimate patient-specific attenuation maps [19]. Such an approach is ob- 
viously not possible for PET, where scattered lines of response are sometimes formed outside the body and/or outside the imaging field of view.

For activity quantification, attenuation and scatter have inverse effects on the activity estimate. That is, uncorrected attenuation will allow too few photons to be detected and, therefore, the activity estimate will be too low. Uncorrected scatter corruption will allow too many photons to be detected and, therefore, the activity estimate will be too high. The tendency in nuclear medicine has been to separately compensate (or correct) for attenuation and for scatter. This tendency can be considered a desirable separation of the compensation problem into two simpler parts. It is analogous to plane-by-plane reconstruction versus 3D reconstruction. However, with the ability to handle bigger computational loads, it has arguably become advantageous to undertake 3D reconstruction in both SPECT and PET. In SPECT, an example is the use of $3 \mathrm{D}$ collimator-detector response information during reconstruction. In PET, the advantage of the $3 \mathrm{D}$ over the $2 \mathrm{D}$ acquisition mode is an increase in the coincidence efficiency by about a factor of 5 even if this is generally accomplished at the expense of increasing the system sensitivity to random and scattered coincidences and the complexity and computational burden of the 3D reconstruction algorithm. So, it can arguably be said that the newer methods of handling scatter and attenuation at the same time have potential advantages over the older separate corrections. The most ambitious of these combined corrections, originally called inverse Monte Carlo [20], attempts to reconstruct scattered photons into their voxel of origin. It will be discussed in slightly more detail below. The approach is considered still to be too ambitious to be practical [21]. More practical approaches simply take into account scatter, as well as attenuation, when the forward projection step of an iterative reconstruction iteration is carried out, but "put back" only unscattered photons.

\section{Modelling the scatter component in uniform and non-uniform media}

We will define modelling the scatter response as creating a representation of the scatter counts in a projection or sinogram that corresponds to a particular activity distribution in the object as well as to a particular distribution of the linear attenuation coefficients or mass density in the object. The current practice of developing theoretical scatter models involves four different stages: characterisation, development, validation and evaluation [22].

1. Characterisation. The scatter response function (srf) is defined as the result of modelling the scatter component for a simple source distribution, such as a point or line. The $s r f$ is studied using a variety of phantom geometries, source locations, scattering medium shapes, sizes and compositions, as well as imaging system-related parameters (an example of the last-mentioned is the detector energy resolution [23]). The goal is to fully understand and characterise the parameters influencing its behaviour.

2. Development. From knowledge and insight gained during the characterisation step, an appropriate scatter model can be developed, often by using the same tools. This model can be a simple one limited to homogeneous attenuating media, or an elaborate one taking into account more complex inhomogeneous media.

3. Validation. The validation step is the crucial part and involves comparisons between either experimental measurements or Monte Carlo simulation studies and predictions of the theoretical model that has been developed. Monte Carlo simulation is generally preferable for practical reasons such as the ease of modelling and because it can separate scattered counts from unscattered counts. Again, this validation can be performed using simple phantom geometries (point and line sources in a uniform elliptical cylinder) or more complicated anthropomorphic phantoms that mimic clinical situations.

4. Evaluation. Obviously, evaluation of the theoretical scatter model with respect to its intended use, i.e. scatter correction, constitutes the last step of the whole process. Assessment of the intrinsic performance of the scatter compensation algorithm that is based on the developed model, as well as its effectiveness in comparison to existing methods, is recommended.

Accurate simulation of scatter in SPECT/PET projection data is computationally extremely demanding for activity distributions in non-uniform dense media. Such simulation requires information about the attenuation map of the patient. A complicating factor is that the scatter response is different for every point in the object to be imaged. Many investigators have used Monte Carlo techniques to study the scatter component or $\operatorname{srf}[17,24$, $25,26,27,28]$. However, even with the use of variance reduction techniques, these simulations require large amounts of computer time. Moreover, the simulation of the $s r f$ for each patient is impractical.

Figure 2 shows the energy pulse-height distribution obtained by simulation of a gamma-emitting $99 \mathrm{mTc}$ line source in the centre of a water-filled cylindrical phantom and a uniform positron-emitting ${ }^{18} \mathrm{~F}$ cylindrical source. The scattered events in the energy pulse-height distribution have been separated according to the order of scattering. It is clear from viewing Fig. 2 that events from some scattered photons will not be rejected by the usual [126-154 keV] and [350-650 keV] energy discrimination, in SPECT and PET, respectively, due to the limited energy resolution. Scattered photons which 
Fig. 2. A An energy spectrum for a gamma-emitting $99 \mathrm{~m} \mathrm{Tc}$ line source on the axis of a water-filled cylinder simulated using the Monte Carlo method. The spectrum due to primary and scattered photons (solid line) is separated into different contributions (total scattering or different orders of photon scattering). The distributions of the various orders of scattered and unscattered photons are shown by broken lines. The experimentally measured spectrum is also shown (dots). B Illustration of the energy distribution due to unscattered and scattered photons resulting from the simulation of a 20-cm-diameter cylinder filled with a uniform positron-emitting ${ }^{18} \mathrm{~F}$ source separated into different contributions (total scattering or different orders of photon scattering). Typical energy acquisition windows for both cases are also shown. (Adapted from [23] and [72])

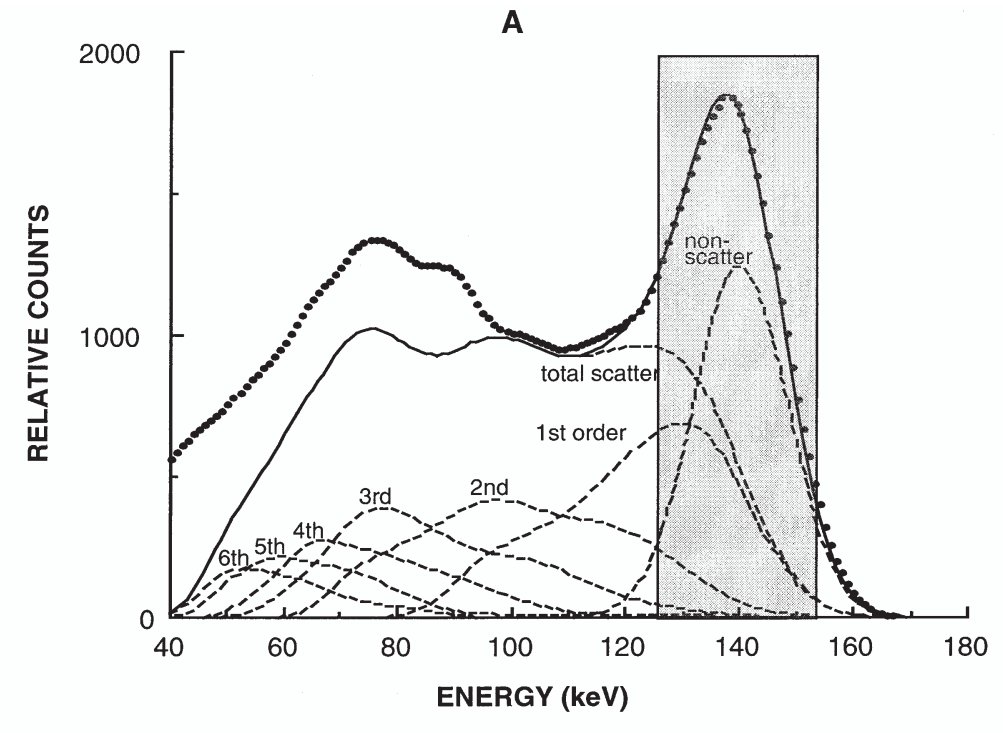

B

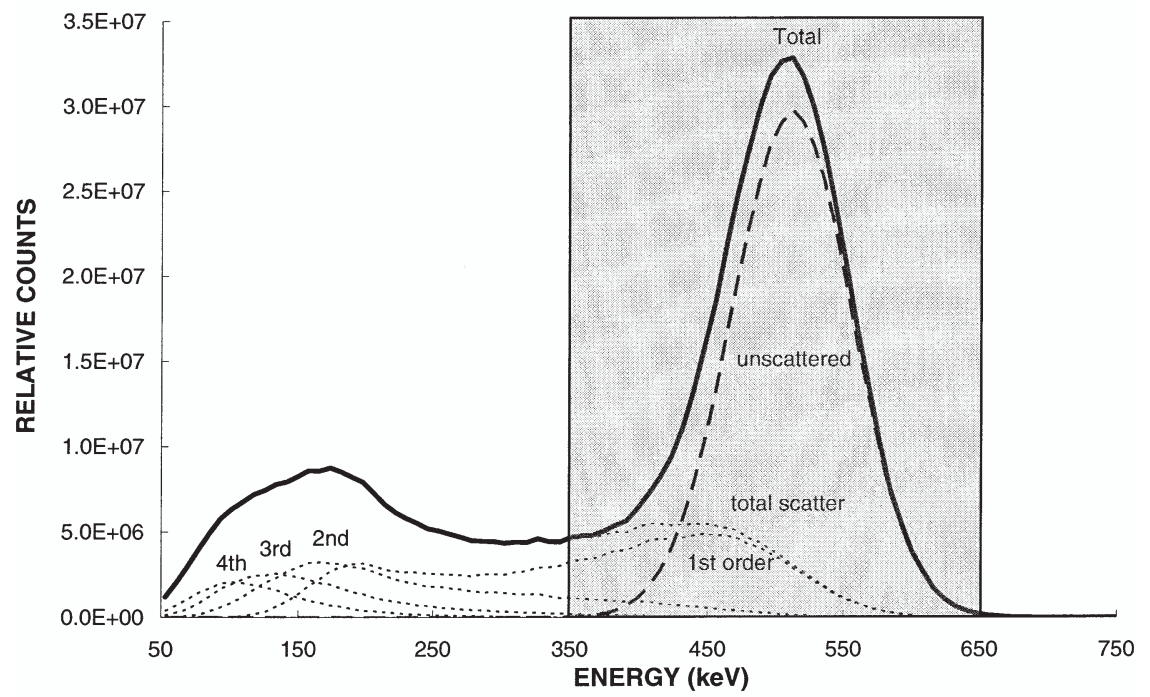

fall within the photopeak window consist mainly of photons which have only scattered once (first order). The lower level threshold (LLT) can be easily changed and its effect on the scatter component studied in an effective way. Figure 3 shows transaxial profiles in the projection space for PET simulations of a line source in a 20 -cm-diameter water-filled cylinder as a function of the LLT. In each case, the centre of the cylinder is located at the origin of the graph. It is clear that the boundary (at $-10 \mathrm{~cm},+10 \mathrm{~cm}$ ) of the object has no influence on the profile. However, the value chosen for the LLT greatly influences the amount of scatter in the projection data.

Scatter is often measured by imaging a line source placed at the centre of a water-filled cylinder. Line spread $\operatorname{srfs}$ (LSFs) are generated and the scatter fraction $(S F)$ determined by fitting the scatter tails of the LSFs to a mono-exponential function. The scatter fraction is defined as scatter divided by total counts recorded, where total and scatter are calculated as the integral of the LSF and the fit to the tails, respectively. The variation of the scatter fraction was investigated for a line source located at the centre of a uniform cylindrical phantom as a function of its size and for three lower energy thresholds $(250,380$ and $450 \mathrm{keV})$. The second part of Fig. 3 shows the scatter fraction estimated directly from the results of the Monte Carlo simulation [29] where the simulated PET scanner operating in 3D mode has an axial field of view of $16.2 \mathrm{~cm}$ and an energy resolution of $23 \%$ for 511-keV photons.

Adam et al. used Monte Carlo simulations to study scatter contribution from outside the field of view and the spatial characteristics of scatter for various phantoms [17]. It was concluded that the spatial distribution of multiple scatter is quite different from the simple scatter component and that this fact precludes the rescaling of the latter to take into account the effect of the former for scatter correction purposes. 
A

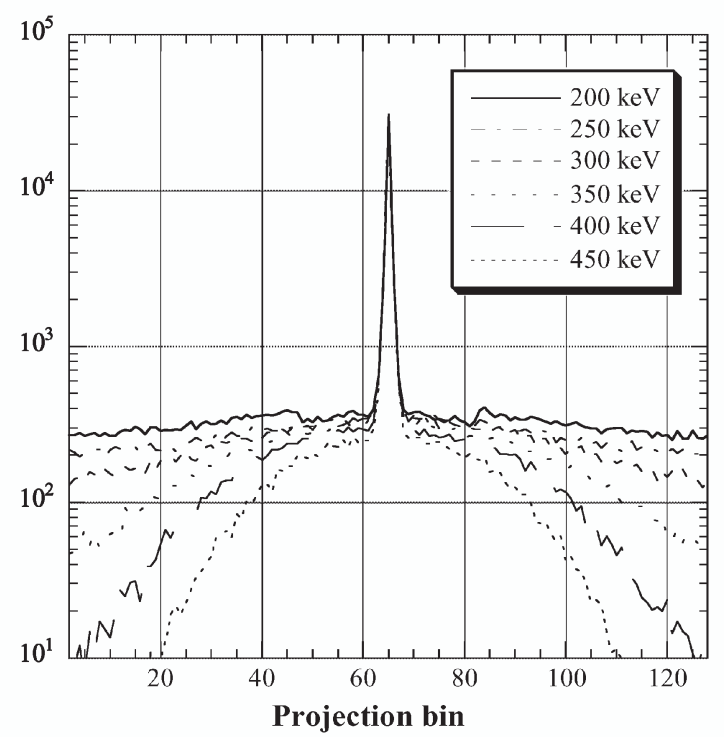

B

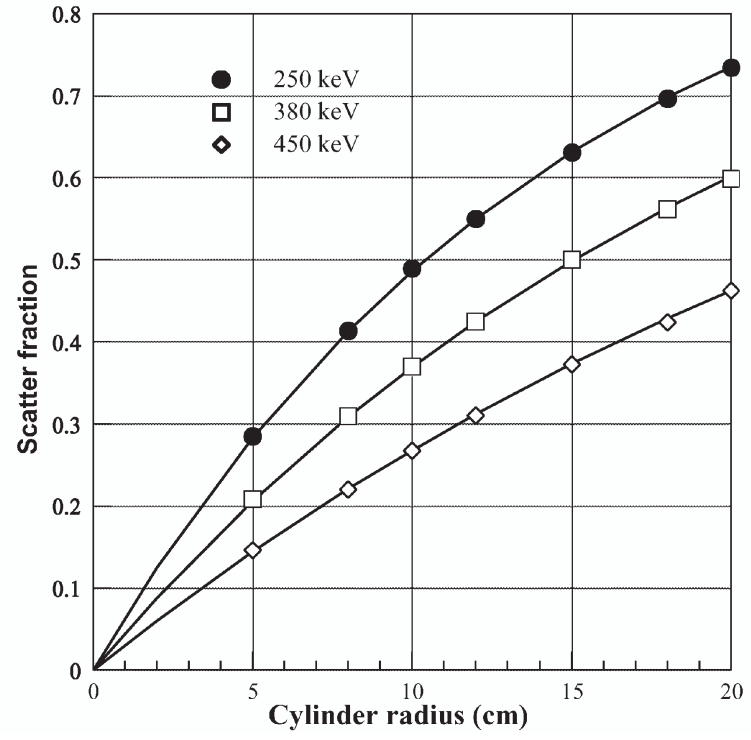

Fig. 3. A Sum of one-dimensional transaxial projections resulting from the simulation of a line source placed in a 20-cm-diameter cylinder filled with water as a function of the lower energy discrimination threshold. This illustrates the compromise that should be attained between increasing the lower level threshold to reduce scattered events and the variance in the reconstructed images resulting from limited statistics. B Monte Carlo calculations of the variation of the scatter fraction as a function of the radius $R$ of the cylindrical phantom for a central line source using three different LLT settings. The fitted curves are also shown

Analytical scatter models, based on integration of the Klein-Nishina (K-N) equation [30, 31, 32], have practical disadvantages, which are similar to those of Monte Carlo-based methods [33, 34, 35].

One class of methods, which estimates anatomy-dependent scatter, first calculates and stores in tables the scatter responses of point sources behind slabs for a range of thicknesses, and then tunes these responses to various object shapes with uniform density [36]. This method is referred to as slab-derived scatter estimation (SDSE). A table occupying only a few Mbytes of memory is sufficient to represent this scatter model for fully 3D SPECT reconstruction [37]. A fully 3D reconstruction of a ${ }^{99 m}$ Tc cardiac study based on SDSE can be performed in only a few minutes on a state-of-the-art single-processor workstation. A disadvantage of SDSE compared with matrices generated by Monte Carlo simulation or KleinNishina integration is that it cannot accurately include the effects of the non-uniform attenuation map of the emitting object density.

A few rough adaptations have been proposed to improve the accuracy and computational speed of this method [38, 39, 40] or other similar approaches [41] in non-uniform objects. SDSE has also been modified to be applicable to ${ }^{201} \mathrm{Tl}$ and to non-uniform attenuators such as found in the chest by its authors. The new approach [42] is called effective source scatter estimation (ESSE). It can perhaps be best described briefly by quoting from the authors" abstract: "The method requires 3 image space convolutions and an attenuated projection for each viewing angle. Implementation in a projector-backprojector pair for use with an iterative reconstruction algorithm would require 2 image space Fourier transforms and 6 image space inverse Fourier transforms per iteration."

For implementation, after the effective source of the method's name is generated: "An attenuated projector that models the distance-dependent collimator-detector response blurring was then applied to this effective source to give the scatter estimate." The authors further say: "We observed good agreement between scatter response functions and projection data estimated using this new model compared to those obtained using Monte Carlo simulations."

Beekman et al. [43] reported an accurate method for transforming the response of a distribution in a uniform object into the response of the same distribution in a non-uniform object. However, the time needed to calculate correction maps for transforming a response from uniform to non-uniform objects may be too long for routine clinical implementation in iterative reconstructionbased scatter correction, especially when the correction maps are calculated for all projection angles and each iteration anew. The use of only one order of scatter was sufficient for an accurate calculation of the correction factors needed to transform the scatter response. Since the computation time typically increases linearly with the number of scatter orders, this transformation method yields much shorter computation times than those with straightforward Monte Carlo simulation. The method was also extended to simulate downscatter through nonuniform media in dual-isotope ${ }^{201} \mathrm{Tl} / 99 \mathrm{~m}$ Tc SPECT imaging [44]. 


\section{Scatter correction techniques in SPECT}

The older SPECT scatter correction techniques have been reviewed frequently [3, 45, 46] and many of the newer ones have been included in the newer reviews $[9,47]$. Therefore, rather than giving each correction method the same amount of space, we are going to neglect some older methods covered in the reviews cited and will present long descriptions only for the newer methods and/or for those not extensively covered in the reviews cited. In addition to describing correction methods, we will discuss comparisons of one method to another, and review studies that present evidence of the clinical impact of scatter correction.

\section{Implicit methods}

Scattered photons degrade the point-spread function (PSF) of the SPECT camera; the long tails of the PSF are mainly due to scatter. Thus, deconvolution methods, which correct the images for the PSF, will also implicitly correct for scatter. In general, the PSF will act as a lowpass filter. Deconvolution will restore the high-frequency contents. However, not only the high frequencies in the signal are restored, but also the high-frequency noise is amplified, which in turn can degrade the image. Therefore, the restoration filter is often combined with a lowpass filter that balances that image improvement by deconvolution and its degradation due to amplification of noise. Well-known examples of these filters are the Wiener and Metz filters. Several investigators have analysed these filters in nuclear imaging and compared their performance relative to each other and relative to other filters and scatter correction approaches [48, 49].

\section{Methods requiring a transmission measurement}

The transmission-dependent convolution subtraction (TDCS) method was introduced in 1994 [50]. It was developed for ${ }^{99 \mathrm{~m}} \mathrm{Tc}$ [51] and ${ }^{201} \mathrm{Tl}$ [52]. As described in an earlier review [9], "It draws upon earlier approaches, and is basically an iterative procedure although sometimes only one iteration is used. It also takes the geometric mean of conjugate views, relies on a convolution, uses a ratio of scattered events divided by total events, $S F(x, y)$, and employs a depth-dependent build-up factor, $B(d)$. The $S F(x, y)$ and the $B(d)$ are both variable across the two-dimensional projection image". The basic equation is:

$f^{n}(x, y)=f_{\text {obs }}(x, y)-S F(x, y) *\left[f^{n-1}(x, y) \otimes \operatorname{srf}(x, y)\right]$

Here, $f^{n}(x, y)$ is the scatter corrected emission projection data after the $n^{\text {th }}$ iteration, $f_{o b s}(x, y)$ is the observed photopeak projection data without scatter correction, $f^{n-1}(x, y)$ is the scatter corrected projection data after the $(n-1)^{\text {th }}$ iteration and $x$ is a transverse coordinate while $y$ is an axial one. The two-dimensional convolution operation is performed in projection space after taking the geometric mean and the $\operatorname{srf}(x, y)$ is radially symmetrical and was originally assumed to be an exponential [50]. The $S F(x, y)$ is defined in terms of $B(d)$, which is itself expressed in terms of measured parameters, $A, \alpha$ and $\beta$, and the narrow-beam transmission function, $T(x, y)$ :

$$
S F(x, y)=1-\frac{1}{A-\alpha * T(x, y) \beta_{/ 2}}
$$

Narita et al. used ten iterations whereas only one was originally employed [51]. They also made several small changes by using a scatter function that was the sum of an exponential plus a Gaussian, and by averaging the dependence of $S F$ on the transmission factor from two empirical cases. Kim et al. also modified the original method when they wanted to use it for ${ }^{123}$ I brain imaging [53]. In their study, the original equation for $S F$ as a function of transmission was modified to include a constant additive term. This term was needed to account for septal penetration of a small percentage of photons from ${ }^{123}$ I that have energies greater than $500 \mathrm{keV}$ [53]. They carried out studies of a phantom and of six patients.

The need for more than one iteration in the TDCS method comes about because originally an image reconstructed from the observed projections is used to generate the scatter correction image whereas the true scatterfree image theoretically would give the correct answer [9]. Moreover, in a recent note it has been argued that in addition to using multiple iterations, a matrix of $S P$ values should replace the matrix of $S F$ values as the image approaches the scatter-free image [54]. The authors of the note carried out a test of their suggestion by simulating a $99 \mathrm{~m} \mathrm{Tc}$ point source centrally positioned in a rectangular water phantom of dimensions $20 \times 20 \times 20 \mathrm{~cm}^{3}$. They used an exponential shape for the scatter kernel. They found a better result by using the $S F$ value only for the first iteration and then the $S P$ value for the succeeding nine iterations, compared with using the $S F$ value for either only one iteration or for all ten iterations.

\section{Multiple-energy window (spectral-analytic) approaches}

The multi-energy window approaches include the dualenergy window (DEW) method, the split-photopeak window method, the triple-energy window (TEW) approach, the spectral fitting method, a multi-window method with weights optimised for a specific task and the neuralnetwork methods. The first involves a window usually of equal width to the photopeak window, placed at a lower energy immediately abutting the photopeak [18]. The second involves splitting the photopeak into two equal halves and using information from the relative number of counts in each half [55]. The TEW method [56] uses the photopeak window and two narrower windows, one 
higher and one lower in energy. The spectral fitting method [57] involves establishing the shape of the energy spectrum for unscattered counts (the scatter-free spectrum). The full energy spectrum, which must be measured over some energy range for each pixel (or "superpixel"), usually by list-mode acquisition, is then assumed to be a value times that scatter-free spectrum plus a spectrum for the scattered photons. For each pixel, the multiplicative value and the spectrum for the scattered photons are obtained by finding the least squares fit between the measured composite spectrum and the assumed components. Variations on the basic approach exist [58].

In the task-specific multiple-window method, weights for each energy window are determined using an optimisation procedure $[1,59]$. In the case of brain imaging

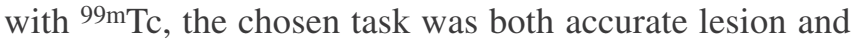
non-lesion activity concentration. The resultant weights had both positive and negative values. In operation, they are combined with the measured spectrum to produce the estimate of total primary counts [1].

A multi-window approach that employs training is scatter estimation using artificial neural networks. These were introduced for scatter correction in 1993 [60]. The reader is referred to that study, to newer studies [61, 62] and to a review [9] for details because the approach cannot be described in a few words.

\section{Approaches requiring iterative reconstruction}

In emission tomography, the scatter estimate can be either precomputed and simply used during iterative reconstruction or generated as well as used during iterative reconstruction [63]. By the former we refer to not explicitly subtracting the scatter estimate from the observed projection data, but simply including it in the statistical model [64]. That is, the goal of a given iteration becomes finding the object that, when it is forward projected and the scatter estimate is added, best fits the measured projection data, with "fit" quantified by the loglikelihood of the Poisson statistical model. In that model, the variance equals the mean, and the mean includes both the unscattered and the scattered contributions.

One class of correction methods uses Monte Carlo simulations $[20,21]$ to compute the complete transition matrix $\left(a_{i j}\right)$, including scatter events. This matrix represents the mapping from the activity distribution onto the projections. Since the first guess of the activity distribution is unlikely to be right, no matter how it is derived, the approach is iterative. Monte Carlo simulation can readily handle complex activity distributions and nonuniform media. Unfortunately, a large amount of memory is required to store the complete non-sparse transition matrix when the fully 3D Monte Carlo matrix approach is used, and without approximations it can take several weeks to generate the full matrix on a state-of-the-art workstation. In addition, the procedure has to be repeated for each patient.

Another class of methods improves the efficiency by utilising a dual matrix approach in which scatter is incorporated in the forward projection step only of an iterative reconstruction algorithm such as the maximum-likelihood expectation-maximisation (ML-EM) or its accelerated version, the ordered-subsets expectation-maximisation (OS-EM) [65].

One of the requirements of this method is the computation of the $s r f$ at each point in the attenuator for all projection views and for each iteration. To avoid slow computation, the correction factors could be calculated only once or alternatively a few times only, given that the calculated scatter component does not change much after the first few iterations of accelerated OS-EM statistical reconstruction have been carried out [43]. Thus, the scatter estimate can be kept as a constant term in either all or only later iterations instead of modifying the scatter estimate in each iteration [40, 64]. In this way, a constant pre-calculated scatter component (using one of the methods described above) can be introduced in the denominator, i.e. the forward projection step of the ML-EM equation:

$f_{j}^{n}=\frac{f_{j}^{n-1}}{\sum_{i} a_{i j}} \sum_{i} a_{i j} \frac{p_{i}}{\sum_{j} a_{i j} f_{j}^{n-1}+\hat{s}}$

where $p_{i}$ and $f_{j}$ are the discrete set of projection pixel values and counts originating from the object voxel activity concentration, respectively, and $(\hat{s})$ is the scatter estimated on all projections.

Interest in this type of approach has been revived with the development of a computationally efficient approach to preserve the main advantages of iterative reconstruction while achieving a high accuracy through modelling the scatter component in the projector using Monte Carlobased calculation of low-noise scatter projections of extended distributions, thus completely avoiding the need for massive transition matrix storage [35].

\section{Impact of scatter correction on clinical SPECT imaging}

At the time of the review by Buvat et al. in 1994, their opinion was that the most clinically used scatter correction method in SPECT was employment of a decreased attenuation coefficient; that is, to not increase reconstructed strength sufficiently during attenuation correction, so as to compensate for not carrying out a reduction in strength to compensate for inclusion of scattered counts. One of the reasons they gave for use of this inferior approach was as follows: "Although most methods have been assessed using simulated and physical data, none has yet faced an extensive procedure of clinical assessment" [3]. However, from the studies cited below in 
Fig. 4. Plot of rCBF assessed by ${ }^{123}$ I-IMP SPECT versus that measured by the gold-standard, ${ }^{15} \mathrm{O}$-water PET imaging. On the left, the SPECT values are without scatter correction. On the right, the SPECT values are with TDCS scatter correction. Each value corresponds to the average over six patients for a particular region of interest in the brain. (Reprinted with permission from [68])

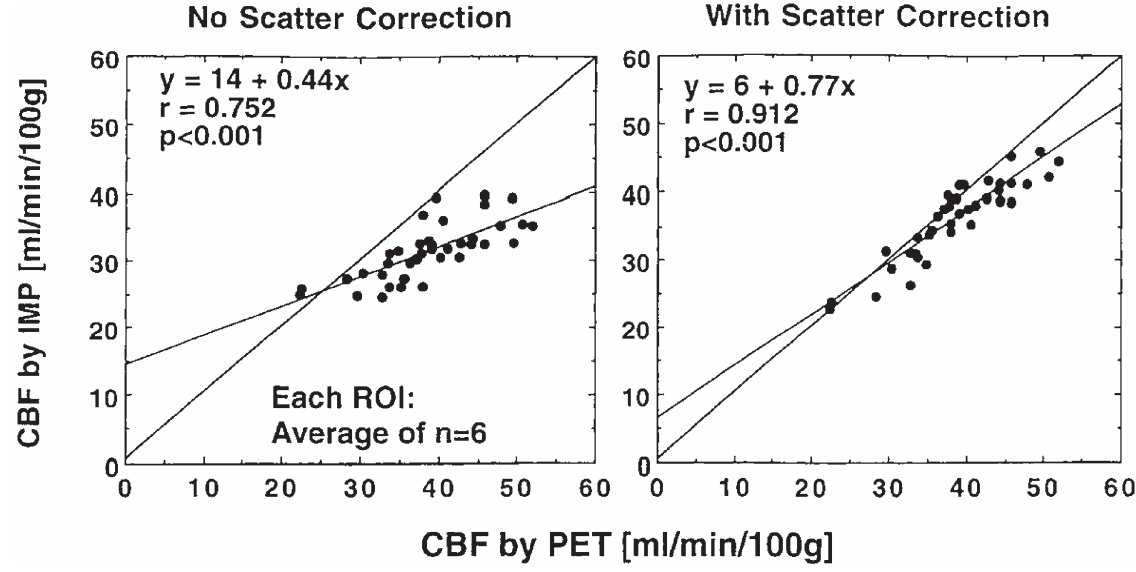

this section, it arguably appears that at least a start on the extensive procedure of clinical assessment has recently been made.

Since the comment by Buvat et al. in 1994, other methods, but still straightforward ones, particularly the DEW method and the TEW method, have become the ones most used in SPECT imaging of patients. For example, Narayanan et al. [66] say the TEW method is their "current clinical standard," and Koral et al. use TEW correction in their clinical SPECT assessment of tumour activity [67]. However, the DEW method is known to be wrong when it uses a spatially invariant value for the ratio of the corrupting scatter counts in the photopeak window to the total counts in the lower-energy monitor window, the $k$ factor [47]. In addition, the narrow monitoring windows in the TEW approach are suspected of generating noisy estimates of the scatter correction image. So, the question is: Can any method prove itself so much better as to displace these, older, simpler methods, or will they continue to be used despite their known or possible shortcomings? Probably the answer will lie in how much effort is devoted to establishing a newer and/or more complicated method in the future.

To date, the TDCS method has been tested for brain and heart imaging [51, 52]. In ${ }^{123}$ I brain imaging, Kim et al. [53] found that their version of the TDCS provided "an acceptable accuracy" in the estimation of the activity of the striatum and of the occipital lobe background. Moreover, parameter values averaged over six collimators from three different SPECT cameras yielded "minimal differences" among the collimators, so new users might not have to calibrate their collimator-camera system.

In a study by Iida et al., it was conclusively found that the regional cerebral blood flow (rCBF) from ${ }^{123} \mathrm{I}-$ iodoamphetamine (IMP) SPECT imaging correlated better with the values from ${ }^{15} \mathrm{O}$-water PET imaging when TDCS scatter correction was employed than when no scatter correction was performed [68]. This fact is shown in Fig. 4 reproduced from the study. The correlation co- efficient is 0.912 with correction but only 0.752 without. Also, the slope of the best-fit line is 0.77 with correction but only 0.44 without.

In SPECT cardiology, Narayanan et al. carried out a human observer study using clinical data from $100 \mathrm{pa}-$ tients undergoing $99 \mathrm{mTc}$-sestamibi perfusion studies [66]. Three cardiology fellows were trained and provided the raw data for the receiver-operating-characteristics (ROC) study. Two methods of scatter correction were separately tested in combination with attenuation correction and detector-collimator response compensation. They were compared with filtered backprojection. Five iterations of OS-EM using 15 subsets were employed for reconstructions with scatter correction, and the scatter estimate was used during the reconstruction rather than subtracted from the projection data (see the section on "Approaches requiring iterative reconstruction" for further explanation of this type of procedure). There are more comments on the two methods in the section on comparison of corrections below. Both methods provided statistically significant improvement for the overall detection of coronary artery disease compared with filtered backprojection. Both also provided larger areas under the ROC curve for localisation of a perfusion defect to the left anterior descending (LAD) territory, to the left circumflex territory (LCx) and to the right coronary artery (RCA) territory. The improvement was statistically significant for the LAD and LCx territories. Significance was computed using the "two-way ANOVA test for statistical significance, followed by Scheffe's multiple comparisons test" using the usual limit of 5\%. This study is impressive to the authors of this review. The only qualification that perhaps needs to be made is that only 55 of the 100 patients had cardiac catheterisation. The remaining 45 subjects were deemed to have a $\leq 5 \%$ likelihood for CAD. Not being experts in the cardiac area, we do not know how reasonable this $5 \%$ likelihood is. Also, we did not see a justification for it in the article, although it probably rests on the clinical assessment that resulted in the patients not being sent for cardiac catheterisation. 


\section{Scatter correction techniques in PET}

Unlike the scatter correction strategies employed in SPECT, those used in PET have been discussed only briefly $[15,69,70]$ with the exception of the extensive reviews provided in book chapters $[9,71,72]$. Over the past two decades, many methods have been developed for the purpose of reducing the degradation of image contrast and loss of quantitative accuracy in PET due to scattered events. The main difference among the correction methods is the way in which the scatter component in the selected energy window is estimated. The most reliable method to determine the actual amount of scatter in the image is accurate modelling of the scatter process to resolve the observed energy spectrum into its unscattered and scattered components. By observing how accurately a scatter correction algorithm estimates the amount and distribution of scatter under conditions where it can be accurately measured or otherwise independently determined, it is possible to optimise scatter correction techniques. A number of scatter correction algorithms for PET have been proposed in the literature. They fall into four broad categories $[15,71]$ :

- Multiple-energy window (spectral-analytic) approaches

- Convolution/deconvolution-based approaches

- Approaches based on direct estimation of scatter distribution

- Statistical reconstruction-based scatter compensation approaches

Different versions of the above methods have been successfully implemented for 3D PET and are briefly discussed below.

\section{Multiple-energy window (spectral-analytic) approaches}

The development of 3D acquisition mode and improvements in the detector energy resolution in PET have allowed the implementation of scatter correction based on the analysis of energy spectra. Several groups investigated the potential of acquiring data in two [73, 74], three [75] and multiple [4] energy windows to develop corrections for scattering in 3D PET. Two variants of the SPECT DEW technique have been proposed for PET: methods estimating the scatter component in the photopeak window from the events recorded in a lower energy window placed just below the photopeak (true DEW) and methods estimating the unscattered component in the photopeak window from the unscattered counts recorded in a high-energy window in the upper portion of the photopeak. The DEW technique of Grootoonk et al. [73] belongs to the former while the estimation of trues method (ETM) [74] belongs to the latter.

The DEW method implemented on the ECAT 953B scanner (CTI/Siemens) assigns detected coincidence events to the upper energy window when both photons deposit energy between $380 \mathrm{keV}$ and $850 \mathrm{keV}$, or to the lower energy window when one or both photons deposit energy between $200 \mathrm{keV}$ and $380 \mathrm{keV}$ [73]. Both energy windows are assumed to contain object scattered and unscattered events. Based on data collected in the two energy windows and scaling parameters derived from measurements of the ratios of counts from line sources due to unscattered (measurements in air) and scattered events (measurements in a head-sized phantom), two equations containing four unknown parameters are solved to estimate the unscattered component in the acquisition energy window.

The ETM method [74] consists in acquiring data simultaneously in two energy windows: a high window with a lower energy threshold higher than $511 \mathrm{keV}$ and a regular acquisition window including the higher window. Therefore, both windows have the same upper level threshold (ULT) value. In the window choice, the method is like the SPECT dual-photopeak window method. The hypothesis of the ETM method is that the number of unscattered coincidences recorded in a given energy range depends on the energy settings of the window and the angle of incidence of the annihilation photons on the detector face. Hence, the unscattered component in the high-energy window can be related to the unscattered coincidences in the standard wider window through a function of the energy settings, the radial position in the sinogram for a given line of response and the axial opening for a given radial position. This calibrating function is assumed to be independent of the source distribution. The unscattered component in the wide energy window can thus be calculated and subsequently subtracted from the data recorded in the regular window to produce a scattered sinogram. The unscattered component in the regular window is then obtained by smoothing that sinogram and subtracting it from the data recorded in the standard window.

The TEW method [75] was suggested as an extension of the DEW technique. Coincidence events are recorded in three windows: two overlapping windows having the same ULT settings (450 keV) and located below the photopeak window and a regular window centred on the photopeak and adjacent to the low windows. A calibrating function that accounts for the distribution of scattered coincidences at low energies is obtained by calculating the ratio of the coincidence events recorded in both low-energy windows for the scanned object and for a homogeneous uniform cylinder. The scatter component in the standard acquisition window is then estimated from the calibrating function and the narrower lowenergy window.

The multispectral method is based on the acquisition of data in a very large number (typically 256) of windows of the same energy width $(16 \times 16$ energy values for the two coincident photons). The spatial distribution of scattered and unscattered components in each window 
can be well fit using simple mono-exponential functions [4]. It has been shown that "while subtraction of object scatter is necessary for contrast enhancement and quantitation accuracy, restoration of detector scatter preserves sensitivity and improves quantitation accuracy by reducing spillover effects in high-resolution PET" [76]. The statistical noise in each window is a potential problem. The hardware and software for multiple-window acquisition remain the major obstacles to implementation of the method on commercial PET scanners.

\section{Convolution-deconvolution based approaches}

Techniques based on convolution or deconvolution estimate the distribution of scatter from the standard photopeak data. The $S F$, which gives an indication of the expected amount of scatter, and the srf, which defines the spatial distribution of scatter, are usually the two parameters that need to be determined a priori. A pure additive model of the imaging system in which the recorded data $\left(p_{o}\right)$ are composed of an unscattered $\left(p_{u}\right)$ and a scattered $\left(p_{s}\right)$ component plus a noise term due to statistical fluctuations is generally assumed. The problem to be addressed consists in estimating $p_{u}$ from $p_{o}$ that is contaminated by scatter, or alternatively estimating $p_{s}$ and then calculating $p_{u}$. The proposed methods differ in the way the $\operatorname{srf}$ is defined.

The convolution-subtraction (CVS) technique developed for 3D PET [77] operates directly on projection data (pre-reconstruction correction). The method is generally based on convolving the source distribution with the srf to obtain an estimate of the scatter component. One makes one of two assumptions: the stationary or the nonstationary assumption. With the stationary assumption, the $\operatorname{srf}$ is assumed to be analytically defined and not dependent on the object, activity distribution, etc. Because this assumption is only approximately correct, an iterative procedure is generally used. The rationale is that with each iteration, the input to the scatter estimation step more closely approximates $p_{u}$. Using a damping factor to prevent oscillations in the result has also been suggested [77]. With the non-stationary assumption, one improves on the previous approximation by taking into consideration the dependence of the srf upon source locations, object size, detector angle, etc. There is a continuing interest in developing the non-stationary CVS scatter correction techniques. Different methods have been proposed in the literature for SPECT [25] and 2D PET imaging [5]; the extension of such models for 3D PET should in principle be straightforward. The CVS approach can also be applied to the reconstructed images (post-reconstruction correction). In this case, the scatter estimates are reconstructed and then subtracted from the non-corrected reconstructed images of the acquired data [78].

The curve-fitting approach is based on the hypothesis that detected events assigned to lines of response outside of the source object must have scattered and that the scatter distribution corresponds to a low-frequency component that is relatively insensitive to the source distribution. Estimation of the unscattered component can thus be performed in three successive steps: (a) fitting the activity outside the source object with an analytical function (e.g. Gaussian), (b) interpolating the fit inside the object and (c) subtracting the scatter component from the observed data [79]. The accuracy of this class of scatter correction methods depends on how accurately the scatter component can be estimated. The appropriate choice of a set of fitting parameters, which should be optimised for each PET scanner and for different distributions of radioactivity and attenuation coefficients, is the dominant factor.

Links et al. [80] studied the use of two-dimensional Fourier filtering to simultaneously increase quantitative recovery and reduce noise. The filter is based on the inversion of the scanner's measured transfer function, coupled with high-frequency roll-off. In phantom studies, they found improvements in both "hot" and "cold" sphere quantification. Fourier-based image restoration filtering is thus capable of improving both accuracy and precision in PET.

\section{Approaches based on direct calculation of scatter distribution}

This class of methods assumes that the distribution of scattered events can be estimated accurately from either the information contained in the emission data or that in both the emission data and the transmission data. For the majority of detected scattered events, only one of the two annihilation photons undergoes a single Compton interaction. The rationale for most methods in this class is that the overall scatter distribution can be computed from the single-scatter distribution $(\sim 75 \%$ of detected scattered events) and that this latter can be scaled to model the distribution of multiple-scattered events [81]. The multiple-scatter distribution is generally modelled as an integral transformation of the single-scatter distribution. Monte Carlo simulation studies of various phantom geometries demonstrated the potential and limitations of this method for fully 3D PET imaging by direct comparison of analytical calculations with Monte Carlo estimates $[17,26]$.

The model-based scatter correction method developed by Ollinger [81] uses a transmission scan, an emission scan, the physics of Compton scatter and a mathematical model of the scanner for use in a forward calculation of the number of single-scatter events. Parameterisation of a fast implementation of this algorithm has recently been reported [82]. The main algorithm difference from that implemented by Ollinger [81] is that "the scatter correction does not explicitly compute scatter for azimuthal angles; rather, it determines 2-D scatter estimates for data 
within 2-D 'super-slices' using as input data from the 3-D direct-plane (non-oblique) slices". A single-scatter simulation (SSS) technique for scatter correction where the mean scatter contribution to the net true coincidence data is estimated by simulating radiation transport through the object was also suggested and validated using human and chest phantom studies [32]. The same author reported on a new numerical implementation of this algorithm, which is faster than the previous implementation, currently requiring less than $30 \mathrm{~s}$ execution time per bed position for an adult thorax [83]. The normalisation problem was solved and multiple scatter partially taken into account. However, the above methods do not correct for scatter from outside the field of view.

Contribution of scatter from outside the FOV remains a challenging issue that needs to be addressed carefully in whole-body imaging, especially with large axial FOV 3D PET scanners. Scatter from outside the field of view can be directly taken into account by acquiring short, auxiliary scans adjacent to the axial volume being investigated. This technique implicitly assumes that the distribution of scatter from outside the FOV has the same shape as that of scatter from inside the FOV. These extra data are naturally available in whole-body imaging. However, this method is impractical for isotopes with a short half-life or rapid uptake relative to the scanning interval. It has also been shown that the attenuation map to be used as input for estimation of the scatter distributions can be derived from magnetic resonance images in brain PET scanning [84]. The contribution of scatter from outside the FOV might be handled effectively using a hybrid approach which combines two scatter correction methods in a complementary way such that one method removes a proportion of scattered events which are not modelled in the second one and vice versa. For example, Ferreira et al. [85] have combined the energy-based ETM algorithm (to remove scatter from outside the FOV) and either CVS or SSS (to remove small-angle scatter) to improve the contrast.

The experimental measurement of the true scatter component is impossible, but it can be accurately estimated using rigorous Monte Carlo simulations. Given a known radioactive source distribution and the density of the object, Monte Carlo techniques allow detected events to be classified into unscattered and scattered events and thus the scatter component to be determined. However, the source and scattering geometry is generally not known in clinical studies. In their Monte Carlo-based scatter correction (MCBSC) method, Levin et al. used filtered backprojection reconstructions to estimate the true source distribution [33]. This input image is then treated as a 3D source intensity distribution for a photontracking simulation. The number of counts in each pixel of the image is assumed to represent the isotope concentration at that location. The image volume planes are then stacked and placed at the desired position in the simulated scanner geometry, assuming a common axis.
The program then follows the history of each photon and its interactions in the scattering medium and traces escaping photons in the block detectors in a simulated 3D PET acquisition. The distributions of scattered and total events are calculated and sorted into their respective sinograms. The unscattered component is equal to the difference between measured data and the scaled and smoothed scattered component. To reduce the calculation time, coarser sampling of the image volume was adopted, assuming that the Compton scatter distribution varies slowly over the object. For obvious reasons, the implemented method does not correct for scatter from outside the field of view and further refinements of the technique were required to take this effect into account. A modified version of this approach was therefore suggested [86]. The data sets were pre-corrected for scatter and the reconstructed images were then used as input to the Monte Carlo simulator [29]. This approach seems reasonable for a more accurate estimation of the true source distribution. Faster implementations of similar approaches have also been described elsewhere [34].

\section{Iterative reconstruction-based scatter correction approaches}

Development of scatter models that can be incorporated into statistical reconstruction such as OS-EM for PET continues to be appealing; however, implementation must be efficient to be clinically applicable. It is worthwhile to point out that, with few exceptions [28, 87, 88], most of the research performed in this field is related to SPECT imaging as reported previously. In the study by Werling et al. [88], the preliminary results obtained using a fast implementation of the SSS algorithm [83] were not satisfactory, and thus spurred further research to incorporate a more accurate model that took into account multiple scatters. Further development and validation of this class of algorithms in whole-body 3D PET are still needed.

Another technique for scatter correction in 3D PET, called statistical reconstruction-based scatter correction, was also recently proposed [28]. The method is based on two hypotheses: (a) the scatter distribution consists mainly of a low-frequency component in the image, (b) the low-frequency components will converge faster than the high-frequency ones in successive iterations of statistical reconstruction methods. This non-uniform convergence property is further emphasised and demonstrated by Fourier analysis of the ML-EM algorithm [89] and successive iterations of inverse Monte Carlo-based reconstructions [20]. The low-frequency image is estimated using one iteration of the OS-EM algorithm. A single iteration of this algorithm resulted in similar or better performance than four iterations of the CVS method [86]. 
Fig. 5. Examples of clinical PET images reconstructed without (left) and with (right) scatter correction for typical ${ }^{18}$ F-FDG brain (A) and wholebody (transaxial and coronal slices) (B) scanning. Note that scatter correction improves the contrast between the grey matter, the white matter and the ventricles in brain imaging and removes background counts in the abdomen and lungs in myocardial imaging. The myocardium is also better delineated after scatter correction. The brain images were corrected for attenuation and reconstructed using an analytical 3DRP algorithm while the whole-body images were reconstructed using normalised attenuation weighted, OS-EM iterative reconstruction (two iterations, eight subsets) followed by post-processing Gaussian filter $(\mathrm{FWHM}=6.0 \mathrm{~mm})$
A

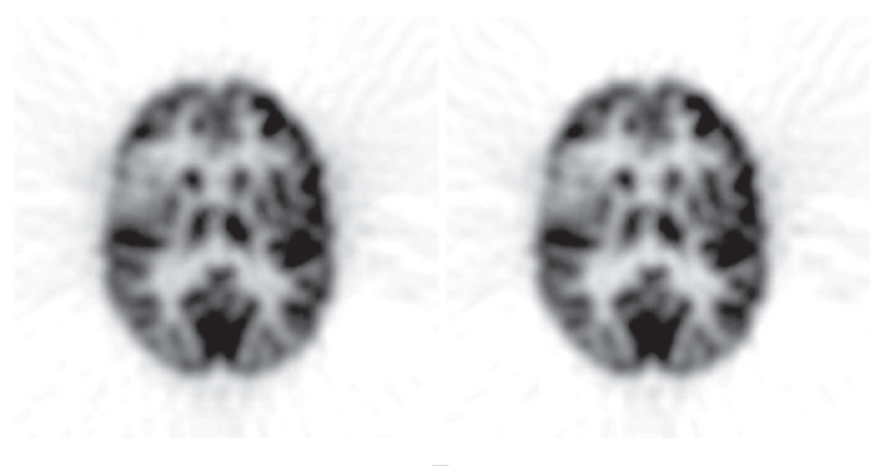

B

\section{Impact of scatter correction on clinical PET imaging}

There is little in the literature reporting systematic studies on the clinical impact of different scatter correction techniques versus no correction in 3D PET. It is well known that subtraction-based scatter correction increases statistical noise. However, in general scatter correction improves the contrast compared with the case where no correction is applied. In particular, the low-count regions and structures are better recovered after scatter compen- sation. Figure 5 illustrates typical clinical ${ }^{18} \mathrm{~F}-\mathrm{FDG}$ brain and thoracic PET scans reconstructed without and with scatter correction, respectively. The data were acquired on the continuously rotating partial-ring ECAT ART tomograph (CTI/Siemens) and corrected for attenuation using collimated ${ }^{137} \mathrm{Cs}$ point source-based transmission scanning. Scatter correction improves the contrast between the different brain tissues and removes background counts in the abdomen and lungs. The myocardium is also better delineated after scatter compensation. Thus, there is consensus within the nuclear medicine 
community with respect to the potential usefulness and necessity of scatter correction for either qualitative interpretation of patient images or extraction of clinically useful quantitative parameters. The main application which is still the subject of debate is ${ }^{15} \mathrm{O}\left[\mathrm{H}_{2} \mathrm{O}\right]$ brain activation studies characterised by low-count imaging protocols, where scatter subtraction might jeopardise the power of statistical analysis significance. In these cases, PET studies focus on identification of functional differences between subjects scanned under different conditions. Whether the scatter component can be considered as constant between the two conditions for inter-subject comparisons still needs to be demonstrated. This constancy is required to confirm the hypothesis that the outcome of statistical analysis (reflecting subtle changes in distribution of radiotracer) does not change greatly with and without scatter compensation.

In radiotracer modelling studies, differences of $10-30 \%$ in the kinetic parameters derived from patient studies are often found to be significant [79]. The magnitude of the scatter correction may cause some parameter values to increase several-fold, with an associated increase in noise. Application of such a correction, if the increase in noise cannot be prevented, would jeopardise the ability to detect subtle biological effects.

\section{Evaluation of scatter correction approaches}

\section{Comparison of methods}

In either SPECT or PET, it has been difficult to establish the superiority of one method over another. The main difference between the correction methods is the way in which the scatter component in the selected energy window is estimated. A limited number of studies reported the comparative evaluation of different scatter correction methods in both SPECT [37, 90, 91, 92] and PET [86, $93,94]$ imaging. There is no single figure of merit that summarises algorithm performance, since performance ultimately depends on the diagnostic task being performed. Well-established figures of merit known to have a large influence on many types of task performance are generally used to assess image quality [95]. Many papers dealing with the evaluation of scatter correction techniques compare relative concentrations within different compartments of a given phantom with the background compartment serving as a reference. This approach possibly obscures what is actually going on, does not necessarily reflect the accuracy of the correction procedure and might bias the evaluation procedure [71]. Therefore attempts should be made to evaluate results in absolute terms.

Ljungberg et al. looked at four methods for scatter correction with ${ }^{99 \mathrm{~m} T c}[90]$. They were the DEW method, the split-photopeak method, a version of the TEW method where only two windows are actually employed and a method based on scatter line-spread functions. The testing involved a brain phantom and employed Monte Carlo simulation. The results indicated "...that the differences in performance between different types of scatter correction technique are minimal for Tc-99m brain perfusion imaging." Thus, in 1994 using filtered backprojection reconstruction with a pre- and post-filter, comparing four particular methods, the conclusion was that there was no difference.

Buvat et al. examined nine spectral or multi-energy window methods, again for $99 \mathrm{mTc}$. They simulated a single, complex phantom [91]. The authors made a considerable number of detailed observations in comparing the nine different scatter compensation methods, some of which were closely related. They also made the important distinction of judging the methods based on relative quantification and on absolute quantification. Based on relative quantification, the TEW approach, simplified for $99 \mathrm{mTc}$, and two factor analysis methods yielded the best results. However, the same methods were not the best based on absolute quantification. That is, in a plot where each value pair represented the estimated and true number of scattered counts in a pixel, the DEW correction method yielded the result which most closely followed the line of identity. Buvat et al. commented on other considerations, such as a greater need for energy linearity in physical cameras for certain methods. They also came to additional conclusions, not all of which are as transparently justified to the authors of this review as those presented above.

Three studies referred to earlier compared new SPECT scatter correction method with the TEW method. Narita et al. compared results from their version of TDCS with the results from TEW scatter correction and concluded that their method produced a much smoother scatter estimate, and that the resulting signal to noise ratio was better than with TEW correction $[51,52]$.

Iida et al. [68], in the study referred to earlier, compared the TDCS method with the TEW method in the same patients (in both cases, the attenuation correction approach was based on a transmission scan of the patient). The result for $\mathrm{rCBF}$ for one particular patient is shown in Fig. 6, reproduced from their study. The authors state: "The increased image noise in the TEW corrected images is clearly apparent." We basically agree with the authors but would qualify the statement by pointing out that what is most striking to us in the comparison is how closely the results are the same. Also, in one part of the authors' work they carried out a detailed comparison between TDCS and TEW while holding the method of attenuation correction constant. Table 2 of the publication presents the $\mathrm{rCBF}$ in $\mathrm{ml} / \mathrm{min} / 100 \mathrm{~g}$ from the IMP SPECT as a function of the method for 39 regions of interest distributed throughout the brain. The mean of the $\mathrm{rCBF}$ and the standard deviation about that mean are given for the six patients evaluated. When one examines these data and computes the relative standard deviation, 
Fig. 6. Comparison of rCBF ${ }^{123}$ I-IMP SPECT images for a normal volunteer using TDCS scatter correction or TEW scatter correction. In the words of the authors of the study, "Although images were clearly noisier with TEW, rCBF images were visually in good agreement between the two methods." See the comments of the authors of this review in the "Comparison of methods" subsection. (Reprinted with permission from [68])

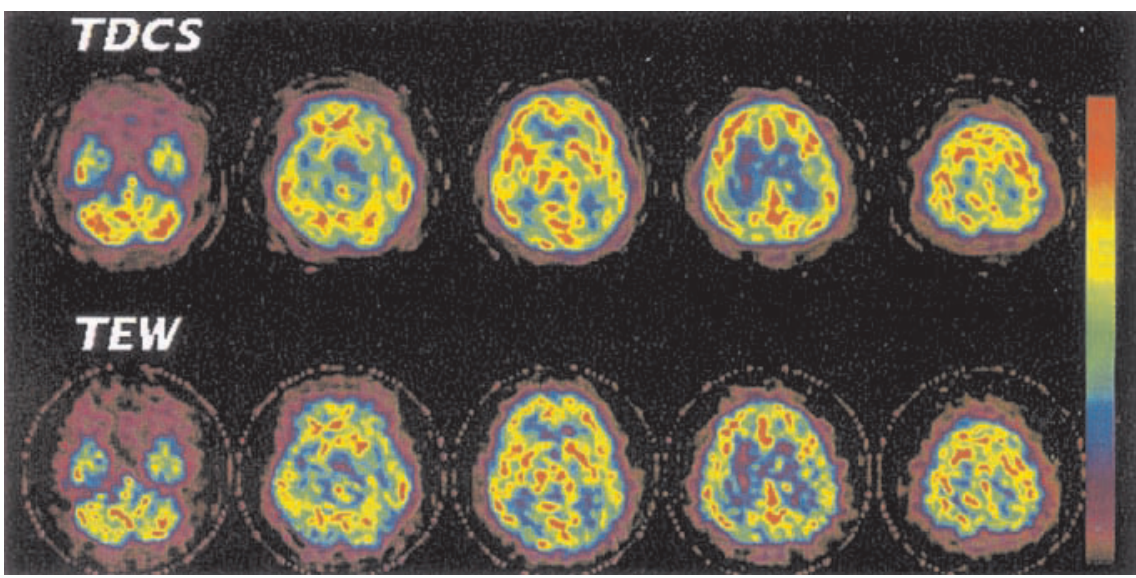

one finds two surprising results: (1) it is smaller with the TEW method than with the TDCS method for 34 out of the 39 regions, and (2) the average value of the relative standard deviation is $21.24 \%$ with the TEW method while it is $24.01 \%$ with the TDCS method. Therefore, it does not appear that the TEW method generates more noise in the quantitative measure of interest. In total, the study by Iida et al. shows that the important matter is to carry out a scatter correction; which scatter correction to use is of secondary importance.

Narayanan et al. compared the ESSE method with the TEW method in the same cardiac patients [66]. The area under the ROC curve was virtually identical for the LCx territory for the two methods, but was larger with ESSE for detection of coronary artery disease for the LAD territory and for the RCA territory. The difference was statistically significant $(P<0.05)$ only for the ROC curves for the LAD territory. The authors of the study added several caveats, but stated: "With TEW-like scatter compensation strategies being easier to implement and having a minimal impact on reconstruction time compared to ESSE, the slightly better performance of ESSE with the clinical images of this study may not be enough to strongly favour its use clinically."

As a footnote to the question of the magnitude of the noise with TEW scatter correction, we note that King et al. [96] have reported that, judged by the normalised mean square error between the estimated and true image, low-pass filtering of the TEW scatter estimate "dramatically decreases" that error, and "inclusion of the scatter estimate directly" into the reconstruction (see the description of such inclusion elsewhere in this paper) also decreases the normalised mean square error compared with simple subtraction. Therefore, it is important for the reader to notice in studies of TEW scatter correction whether and how the scatter estimate is low-pass filtered (or smoothed) and whether it is simply subtracted or is included in the reconstruction.

A recent SPECT study [59] compared compensation for scatter, lead X-rays and high-energy contamination using an artificial neural network approach employing three separate networks with the task-specific multiplewindow method for lesion detection and activity estimation tasks in realistic ${ }^{67} \mathrm{Ga}$ Monte Carlo simulations. The comparison was carried out for each of the three main photopeaks of ${ }^{67} \mathrm{Ga}$. In the section pertaining to activity estimation, the activity amplitude of each of seven spheres was determined, one by one, by a least squares estimation that involved calculating a sum of squared differences, $\chi^{2}$, given by:

$\chi^{2}=\sum_{x, z, \theta}\left[A_{i}\left[f_{i}(x, z, \theta)\right]+B(x, z, \theta)-d_{i}(x, z, \theta)\right]^{2}$

where $i$ is an index that runs over seven spheres, $A_{i}$ is the unknown activity amplitude for the $i^{\text {th }}$ sphere, $f_{i}(x, z, \theta)$ is the projected and blurred shape of the $i^{t h}$ sphere, $B(x, z, \theta)$ is the projection of the background, $d_{i}(x, z, \theta)$ is the projection of the $i^{\text {th }}$ sphere plus background, $x$ is the transverse spatial coordinate, $z$ is the axial coordinate and $\theta$ is the projection angle. The activity was determined by taking the derivative of $\chi^{2}$ with respect to $A_{i}$, setting that derivative to zero (to find a minimum) and solving for $A_{i}$. The $f_{i}(x, z, \theta)$ and the $B(x . z . \theta)$ for each sphere were from only the primary photons. Then, at first $d_{i}(x, z, \theta)$ was also from only the primary photons; this led to the true value of the activity amplitude. Then, $d_{i}(x, z, \theta)$ was the scatterincluded projection, corrected for scatter by a neural network method; this led to a value of the activity amplitude with that correction. Then, $d_{i}(x, z, \theta)$ was the projection corrected for scatter by a task-specific multi-window method; this led to a value of the activity amplitude with that correction. Moreover, there were 100 realisations for each of the three, and the mean and variance of the activity amplitude were calculated over these realisations for the primaries and for each correction. Next, the average activity amplitude over the realisations for the primaries gave the true activity amplitude; the variance was also computed. Using the true activity amplitude, the bias in the mean activity amplitude for each correction was calculated. Next, the mean square error for the primaries and for each correction was calculated from the mean and variance using the standard formula. Final- 


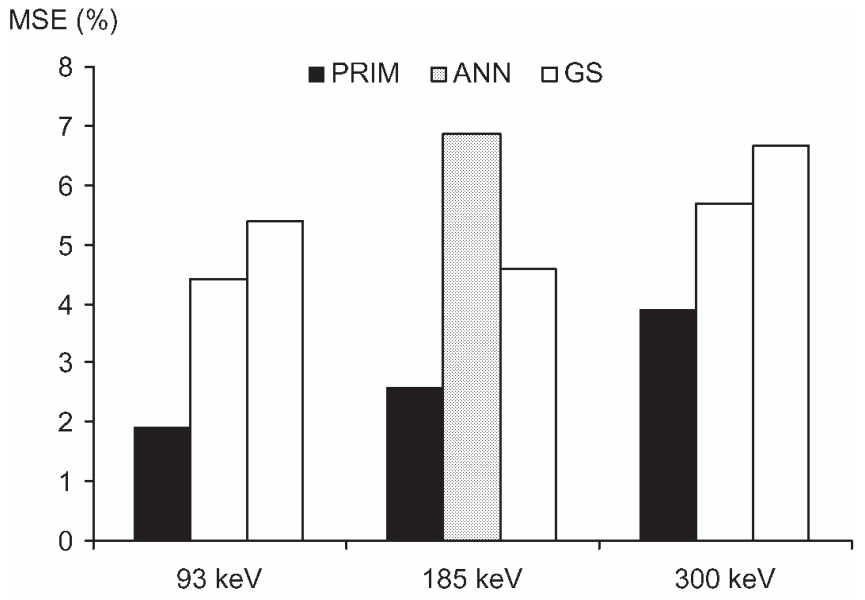

Fig. 7. Mean square error (MSE) of activity estimation. Error was calculated over seven spheres in different phantom locations using either the $93-$, the $185-$, or the $300-\mathrm{keV}^{67} \mathrm{Ga}$ photopeak. PRIM indicates results using Monte Carlo-simulated primaries, which are arguably better than any scatter correction method can achieve. $A N N$ indicates results using all photons after artificial neural network scatter correction, and $G S$ indicates results using all photons after task-specific multiple-window scatter correction. (Reprinted from [59], () 2003 IEEE, with permission)

ly, the mean square error was averaged for the seven spheres for the primaries and for each correction. The result is shown in Fig. 7 [59]. It is seen that the neural network correction method yielded the lower error of the two correction methods for two windows, and the taskspecific multi-window method yielded the lower error for the third window. The result labelled PRIM is for the primaries for which the bias was zero but the non-zero variance gave a non-zero mean-square error. This PRIM mean square error is arguably better than any scatter correction method can do. The closeness of both methods to PRIM, in absolute terms, says that they are accurate and implies that either might perform quite well in a clinical activity quantification task.

Zaidi evaluated five scatter correction techniques in 3D PET using Monte Carlo simulation studies, experimental phantom measurements and clinical studies. The comparison included the DEW technique, the CVS method, two variants of the Monte Carlo-based scatter correction technique and his statistical reconstructionbased scatter correction method [86]. It was concluded that the DEW method showed the best compromise between ease of implementation and quantitative accuracy, but resulted in significant deterioration in the signal to noise ratio. The study was, however, limited to clinical data and phantom studies mimicking brain imaging conditions, suggesting that the conclusions might not be applicable to whole-body imaging.

As a summary for this subsection, most comparative evaluation studies found that all correction methods significantly improved the image quality and contrast compared with the case where no correction was applied.
More importantly, some reports suggested that the differences in the estimated scatter distributions did not have a significant impact on the final quantitative results. Thus, we conclude that at present the important thing is to use some form of correction, although ultimately it may become clear which method is best for a particular application.

\section{Monte Carlo simulations}

Medical imaging simulation tools have proved to be very useful for validation and comparative evaluation of image reconstruction techniques since it is possible to obtain a reference image with which reconstructed images should be compared. Modelling of the imaging process has been improved by more accurate simulation of the physics and instrumentation involved in the process. Monte Carlo software packages, especially those developed specifically for nuclear medicine and with different performance characteristics, have been found useful in the modelling work. Many general-purpose and dedicated Monte Carlo programs have been in use in the field of nuclear imaging, with many of them available in the public domain [97, 98]. Although variance reduction techniques have been developed to reduce computation time, the main drawback of the Monte Carlo method is that it is extremely time-consuming.

The combination of realistic computer phantoms and accurate models of the imaging process allows simulation of nuclear imaging data that are ever closer to actual patient data. Simulation techniques will find an increasingly important role in the future of nuclear medicine research, especially scatter modelling and correction, in light of further development of realistic computer phantoms, accurate modelling of projection data and computer hardware. However, caution must be exercised to avoid errors in the simulation process, and verification via comparison with experimental and patient data is crucial [99]. Interested readers are referred to textbooks cited above that extensively discuss issues related to Monte Carlo modelling in nuclear medicine.

\section{Availability of experimental and simulated phantoms}

Software and physical phantoms used in medical imaging were historically limited to simple point, rod and slab shapes of sources and attenuating media. Such simple geometries are useful in studying fundamental issues of image reconstruction, but clinically realistic distributions cannot be evaluated by such simple geometries. A precise modelling of the human body requires appropriate information on the location, shape, density and elemental composition of the organs or tissues. Several physical and software phantoms modelling different parts of the human body have been developed over the 
years to assess the accuracy of image correction procedures.

Modelling of nuclear imaging is best done with phantom models that match the gross parameters of an individual patient. Recent three- and four-dimensional (dynamic) computer phantoms seek a compromise between ease of use, flexibility and accurate modelling of populations of patient anatomies and of attenuation and scatter properties and biodistributions of radiopharmaceuticals in the patients. It is worth emphasising that even the most sophisticated phantom will never reproduce precisely the human anatomy. Assessment in a preclinical/ clinical setting using animal/human data is, thus, essential.

\section{Clinical, phantom and research studies}

In a clinical environment, evaluation of scatter correction is hampered by the multiplicity of the medical purposes for which the corrections may be studied. There is a clear need for guidelines to evaluate image correction techniques and other image processing issues in emission tomography. Moreover, researchers must be willing to compare clinical results with and without scatter correction, which in most instances doubles the work, rather than simply choosing to use scatter correction or not to use it. For any specific medical task, the evaluation should ideally be based on the performance of human observers. However, this is costly and complex, since a reasonable number of experienced observers should be used to analyse many images under carefully controlled conditions, etc. One severe limitation of performing psychophysical experiments for evaluation of image correction techniques is that it is time consuming and costly.

Another method to assess the effect of scatter is to investigate the errors in tracer kinetic parameter estimation after scatter compensation. Very few papers have addressed this issue during the past decade using SPECT $[100,101]$ and 3D brain PET data $[79,93]$. Further investigations using various SPECT and PET tracers for different clinical situations are necessary to fully characterise the effect of scatter correction on tracer kinetic parameters estimation.

de Vries et al. carried out a study on the effects of SPECT scatter subtraction using DEW on both detection of liver lesions and lesion activity quantification using synthetic $99 \mathrm{~m}$ Tc images [102]. Their conclusion was that the scatter correction could improve the activity quantification but did not help in the detection task.

In SPECT cardiology, Sankaran et al. carried out a human observer study using Monte Carlo data generated for 24 versions of a heart phantom, with a perfusion defect placed in one of six locations [103]. Sixty-four projections over $180^{\circ}$ were simulated for each phantom and four combinations of correction were investigated. They employed the ESSE method for scatter correction. The

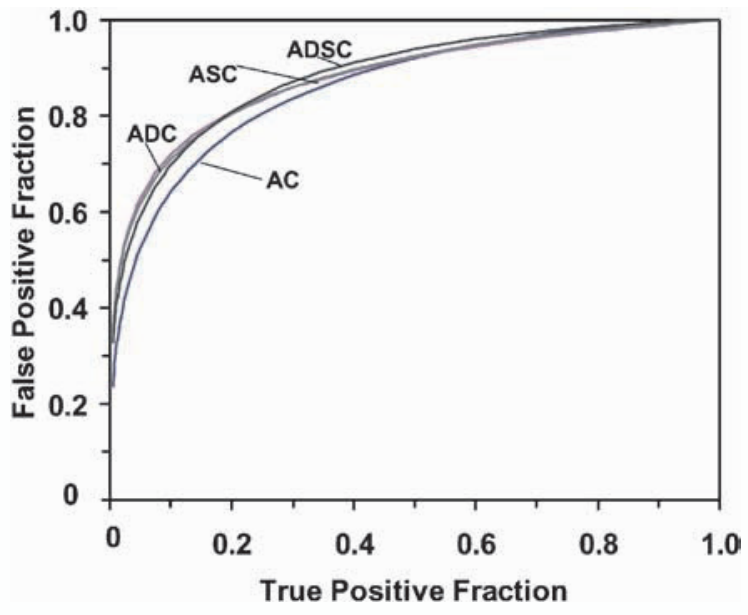

Fig. 8. Average ROC curves using attenuation, scatter, and/or distance-dependent collimator-detector-response compensation. Four combinations are shown. Fitted ROC curves were averaged over five observers. For all methods, six iterations of OS-EM with 16 subsets per iteration and an order 8 Butterworth post-reconstruction filter were used with a cut-off value of 0.16 pixel $^{-1}$. (Reprinted with permission from [103])

area under the average ROC curve with each combination was calculated. The authors found that: "Reconstruction methods that incorporated scatter and detector response compensation had higher indices of detectability than..." attenuation correction alone. The amount of improvement was relatively small (Fig. 8) but was statistically significant using the more generous limit of $10 \%$ rather than $5 \%[P=0.076$ for attenuation and scatter correction (ASC) compared with attenuation correction alone (AC) and $P=0.084$ for attenuation, scatter and collimator-detector response correction (ADSC) versus $\mathrm{AC}]$. In order to carry out the ROC analysis at all with the number of cases they employed, it was necessary to make the statistical noise larger than that typically observed in the clinic.

To clarify the issue of whether scatter correction and iterative reconstruction produces significant changes in ${ }^{18}$ FFDG distribution of reconstructed brain PET images, a recent study aimed at assessing the effect of model-based scatter correction in 3D brain PET studies using statistical parametric mapping (SPM) analysis in healthy volunteers [104]. It was concluded that iterative reconstruction did not result in significant changes, while significant differences in ${ }^{18} \mathrm{~F}-\mathrm{FDG}$ distribution exist when images are reconstructed with and without explicit scatter correction for some cerebral areas. This needs to be acknowledged for adequate interpretation of 3D brain PET images after applying scatter correction. Table 3 lists the peaks of the most significant decreases and increases in brain metabolism when images are normalised using a tracer-specific ${ }^{18} \mathrm{~F}-\mathrm{FDG}$ template. The brain structures were determined from the stereotactic coordinates with respect to the Talairach and Tournoux atlas. Algebraic values of the scores are also shown $(-=$ decrease, $+=$ increase $)$. 
Table 3. Results of statistical parametric mapping analysis comparing cerebral ${ }^{18} \mathrm{~F}-\mathrm{FDG}$ PET images of healthy subjects reconstructed with scatter correction using the single-scatter simulation algorithm with images corrected for attenuation using an effective linear attenuation coefficient without explicit scatter correction and normalised using a tracer-specific template. The stereotactic coordinates corresponding to areas of significant regional decrease (-) and increase $(+)$ in brain metabolism (Z score) in a particular cerebral structure with respect to the Talairach and Tournoux atlas are shown (Adapted from [104])

\begin{tabular}{lrrrr}
\hline \multirow{2}{*}{ Cerebral structure } & \multicolumn{2}{c}{ Stereotactic coordinates $(\mathrm{mm})$} & \multirow{2}{*}{ Z score } \\
\cline { 2 - 4 } & $x$ & \multicolumn{2}{c}{$y$} & \\
\hline Right cerebellum & 26 & -44 & -20 & +6.75 \\
Right thalamus & 8 & -14 & -6 & +6.13 \\
Right middle frontal gyrus & 38 & 38 & 26 & -6.07 \\
Left insula & -34 & -4 & -0 & +5.67 \\
Right medulla & 2 & -34 & -36 & +5.59 \\
Left rectal gyrus & -2 & 34 & -20 & +5.57 \\
Right middle temporal gyrus & 44 & -68 & 22 & -5.43 \\
Right postcentral gyrus & 54 & -26 & 44 & -5.37 \\
Right superior frontal gyrus & 14 & 50 & 28 & -5.26 \\
Left temporal lobe & -50 & -18 & -32 & +5.13 \\
Right inferior frontal gyrus & 22 & 18 & -18 & +5.08 \\
Left precentral gyrus & -44 & -4 & 56 & -4.83 \\
Right superior frontal gyrus & 20 & 50 & -2 & -4.80 \\
\hline
\end{tabular}

\section{Scatter correction in transmission images}

A point that deserves special attention, but which was not discussed previously, is the need for efficient and accurate modelling and correction of scatter in transmission images acquired for attenuation and/or scatter correction purposes, especially when using simultaneous emission/transmission scanning [105]. Some studies have reported the use of model-based approaches to scatter estimation in transmission imaging [106, 107]. Similar approaches are also being developed for X-ray CT imaging [108], which is desirable on dual-modality imaging systems offering the possibility to determine an appropriately scaled patient-specific attenuation map for correcting the emission data. Further studies are still needed to fully characterise the scatter component in transmission imaging in different clinical situations using different acquisition and processing protocols.

\section{Summary and future directions}

Various methods for tackling the scatter correction problem have been proposed. Increasingly sophisticated scatter correction procedures are under investigation, particularly those based on accurate scatter models and iterativebased scatter compensation approaches. Monte Carlo simulation provides insight and might in itself offer a practical correction procedure.

It is gratifying to see the progress that scatter correction has made in the past 20 years, from very crude energy-based approaches, through analytical and Monte Carlo modelling, to the recent iterative reconstruction-based scatter correction approaches. Recent developments have been enormous; in particular, improvements have been achieved in accuracy, precision and computational speed, in conjunction with decreased calibration data. The ne- cessity for scatter correction is well understood in research environments. Moreover, scatter correction is now carried out in some clinical settings, even in institutions without extensive physics and computing support. Implementation of validated techniques in commercial software packages would be useful to further attract the interest of the clinical community. This greater interest would in turn lead to increased refinement of scatter correction techniques. It is expected that with the availability of greater computing power in the near future, more complex and ambitious computer-intensive scatter modelling and correction algorithms will increasingly become clinically feasible.

In summary, the scatter correction issue in $3 \mathrm{D}$ SPECT/PET is an area of considerable research interest; many different groups are very active in this field. The nuclear medicine community can look forward to further progress during the next few years.

Acknowledgements. One of the co-authors (H.Z.) acknowledges the support of grant SNSF 3152A0-102143, awarded by the Swiss National Science Foundation. The other (K.F.K.) acknowledges the support of grant R01 CA87955, awarded by the National Cancer Institute, United States Department of Health and Human Services. The contents are solely the responsibility of the authors and do not necessarily represent the official view of the sponsoring agencies.

\section{References}

1. Moore SC, Kijewski MF, Muller SP, Rybicki F, Zimmerman RE. Evaluation of scatter compensation methods by their effects on parameter estimation from SPECT projections. Med Phys 2001; 28:278-287.

2. Gagnon D, Laperriere L, Pouliott N, deVries DJ, Moore SC. Monte Carlo analysis of camera-induced spectral contamination for different primary energies. Phys Med Biol 1992; $37: 1725-1739$. 
3. Buvat I, Benali H, Todd-Pokropek A, Di Paola R. Scatter correction in scintigraphy: the state of the art. Eur J Nucl Med 1994; 21:675-694.

4. Bentourkia M, Msaki P, Cadorette J, Lecomte R. Object and detector scatter-function dependence on energy and position in high resolution PET. IEEE Trans Nucl Sci 1995; 42:11621167.

5. Bentourkia M, Lecomte R. Energy dependence of nonstationary scatter subtraction-restoration in high resolution PET. IEEE Trans Med Imaging 1999; 18:66-73.

6. Evans RD. The atomic nucleus. New York: McGraw-Hill, 1955.

7. Carlsson GA, Carlsson CA, Berggren KF, Ribberfors R. Calculation of scattering cross sections for increased accuracy in diagnostic radiology. I. Energy broadening of Comptonscattered photons. Med Phys 1982; 9:868-879.

8. Hua C-H. Compton imaging system development and performance assessment [PhD thesis]. University of Michigan, USA; 2000.

9. Zaidi H, Koral KF. Scatter correction strategies in emission tomography. In: Zaidi H, ed. Quantitative analysis in nuclear medicine imaging. New York: Kluwer Academic/Plenum Publishers, 2004.

10. de Vries DJ, Moore SC, Zimmerman RE, Mueller SP, Friedland B, Lanza RC. Development and validation of a Monte Carlo simulation of photon transport in an Anger camera. IEEE Trans Med Imaging 1990; 9:430-438.

11. de Vries DJ, King MA, Moore SC. Characterization of spectral and spatial distributions of penetration, scatter and lead X-rays in Ga-67 SPECT. IEEE Nuclear Science Symposium, 1998. Conference Record. 1998; 3:1707-1710.

12. Dewaraja YK, Ljungberg M, Koral KF. Characterization of scatter and penetration using Monte Carlo simulation in ${ }^{131} \mathrm{I}$ imaging. J Nucl Med 2000; 41:123-130.

13. Moore S, de Vries D, Penney B, Müller S, Kijewski M. Design of a collimator for imaging In-111. In: Ljungberg M, Strand S-E, King MA, eds. Monte Carlo calculations in nuclear medicine: applications in diagnostic imaging. Bristol: Institute of Physics Publishing; 1998:183-193.

14. King MA, Tsui BM, Pan TS, Glick SJ, Soares EJ. Attenuation compensation for cardiac single-photon emission computed tomographic imaging. Part 2. Attenuation compensation algorithms. J Nucl Cardiol 1996; 3:55-64.

15. Zaidi H. Scatter modelling and correction strategies in fully 3-D PET. Nucl Med Commun 2001; 22:1181-1184.

16. Schoder H, Erdi YE, Larson SM, Yeung HW. PET/CT: a new imaging technology in nuclear medicine. Eur J Nucl Med Mol Imaging 2003; 30:1419-1437.

17. Adam LE, Karp JS, Brix G. Investigation of scattered radiation in 3D whole-body positron emission tomography using Monte Carlo simulations. Phys Med Biol 1999; 44:2879_ 2895.

18. Jaszczak RJ, Greer KL, Floyd CE, Harris CC, Coleman RE. Improved SPECT quantification using compensation for scattered photons. J Nucl Med 1984; 25:893-900.

19. Pan TS, King MA, Luo DS, Dahlberg ST, Villegas BJ. Estimation of attenuation maps from scatter and photopeak window single photon-emission computed tomographic images of technetium 99m-labeled sestamibi. J Nucl Cardiol 1997; $4: 42-51$

20. Floyd CE, Jaszczak RJ, Greer KL, Coleman RE. Inverse Monte Carlo as a unified reconstruction algorithm for ECT. J Nucl Med 1986; 27:1577-1585.
21. Buvat I, Lazaro D, Breton V. Fully 3D Monte Carlo reconstruction in SPECT: proof of concept and is that worthwhile? Conference proceedings of the VIIth International Meeting on Fully Three-Dimensional Image Reconstruction in Radiology and Nuclear Medicine, 29 June-4 July 2003, Saint-Malo, France. Available on CD-ROM.

22. Ljungberg M. The Monte Carlo method applied in other areas of SPECT imaging. In: Ljungberg M, Strand S-E, King MA, eds. Monte Carlo calculations in nuclear medicine: applications in diagnostic imaging. Bristol: Institute of Physics Publishing; 1998:207-220.

23. Kojima A, Matsumoto M, Takahashi M, Uehara S. Effect of energy resolution on scatter fraction in scintigraphic imaging: Monte Carlo study. Med Phys 1993; 20:1107-1113.

24. Floyd CE, Jaszczak RJ, Harris CC, Coleman RE. Energy and spatial distribution of multiple order Compton scatter in SPECT: a Monte Carlo investigation. Phys Med Biol 1984; 29:1217-1230.

25. Ljungberg M, Strand SE. Scatter and attenuation correction in SPECT using density maps and Monte Carlo simulated scatter functions. J Nucl Med 1990; 31:1560-1567.

26. Barney JS, Rogers JG, Harrop R, Hoverath H. Object shape dependent scatter simulations for PET. IEEE Trans Nucl Sci 1991; 38:719-725.

27. Frey EC, Tsui BMW. Parameterization of the scatter response function in SPECT imaging using Monte Carlo simulation. IEEE Trans Nucl Sci 1990; 37:1308-1315.

28. Zaidi H. Reconstruction-based estimation of the scatter component in positron emission tomography. Ann Nucl Med Sci $2001 ; 14: 161-171$.

29. Zaidi H, Scheurer AH, Morel C. An object-oriented Monte Carlo simulator for 3D positron tomographs. Comput Methods Prog Biomed 1999; 58:133-145.

30. Riauka TA, Hooper HR, Gortel ZW. Experimental and numerical investigation of the 3D SPECT photon detection kernel for non-uniform attenuating media. Phys Med Biol 1996; 41:1167-1189.

31. Jonsson C, Larsson SA. A spatially varying Compton scatter correction for SPECT utilizing the integral Klein-Nishina cross section. Phys Med Biol 2001; 46:1767-1783.

32. Watson CC, Newport D, Casey ME, deKemp A, Beanlands RS, Schmand M. Evaluation of simulation-based scatter correction for 3-D PET cardiac imaging. IEEE Trans Nucl Sci 1997; 44:90-97.

33. Levin CS, Dahlbom M, Hoffman EJ. A Monte Carlo correction for the effect of Compton scattering in 3-D PET brain imaging. IEEE Trans Nucl Sci 1995; 42:11811188 .

34. Holdsworth CH, Levin CS, Janecek M, Dahlbom M, Hoffman EJ. Performance analysis of an improved 3-D PET Monte Carlo simulation and scatter correction. IEEE Trans Nucl Sci 2002; 49:83-89.

35. Beekman FJ, de Jong HW, van Geloven S. Efficient fully 3-D iterative SPECT reconstruction with Monte Carlo-based scatter compensation. IEEE Trans Med Imaging 2002; 21:867-877.

36. Frey EC, Tsui BMW. A practical method for incorporating scatter in a projector-backprojector for accurate scatter compensation in SPECT. IEEE Trans Nucl Sci 1993; 40:11071116.

37. Beekman FJ, Kamphuis C, Frey EC. Scatter compensation methods in 3D iterative SPECT reconstruction: a simulation study. Phys Med Biol 1997; 42:1619-1632. 
38. Frey EC, Tsui BMW. Modeling the scatter response function in inhomogeneous scattering media for SPECT. IEEE Trans Nucl Sci 1994; 41:1585-1593.

39. Beekman FJ, den Harder JM, Viergever MA, van Rijk PP. SPECT scatter modelling in non-uniform attenuating objects. Phys Med Biol 1997; 42:1133-1142.

40. Kadrmas DJ, Frey EC, Karimi SS, Tsui BM. Fast implementations of reconstruction-based scatter compensation in fully 3D SPECT image reconstruction. Phys Med Biol 1998; 43:857873.

41. Wells RG, Celler A, Harrop R. Analytical calculation of photon distributions in SPECT projections. IEEE Trans Nucl Sci 1998; 45:3202-3214.

42. Frey EC, Tsui BMW. A new method for modeling the spatiallyvariant, object-dependent scatter response function in SPECT. IEEE Nuclear Science Symposium, 1996. Conference Record 2:1082-1086.

43. Beekman FJ, de Jong HW, Slijpen ET. Efficient SPECT scatter calculation in non-uniform media using correlated Monte Carlo simulation. Phys Med Biol 1999; 44:N183-N192.

44. de Jong HW, Beekman FJ. Rapid SPECT simulation of downscatter in non-uniform media. Phys Med Biol 2001; 46:621635.

45. Rosenthal MS, Cullom J, Hawkins W, Moore SC, Tsui BM, Yester M. Quantitative SPECT imaging: a review and recommendations by the Focus Committee of the Society of Nuclear Medicine Computer and Instrumentation Council. J Nucl Med 1995; 36:1489-1513.

46. Zaidi H. Quantitative SPECT: recent developments in detector response, attenuation and scatter correction techniques. Phys Med 1996; 12:101-117.

47. Koral KF. Monte Carlo in SPECT scatter correction. In: Ljungberg M, Strand S-E, King MA, eds. Monte Carlo calculations in nuclear medicine: applications in diagnostic imaging. Bristol: Institute of Physics Publishing; 1998:165181.

48. Yanch JC, Flower MA, Webb S. Improved quantification of radionuclide uptake using deconvolution and windowed subtraction techniques for scatter compensation in single photon emission computed tomography. Med Phys 1990; 17:10111022.

49. King MA, Coleman M, Penney BC, Glick SJ. Activity quantitation in SPECT: a study of prereconstruction Metz filtering and use of the scatter degradation factor. Med Phys 1991; 18:184-189.

50. Meikle SR, Hutton BF, Bailey DL. A transmission-dependent method for scatter correction in SPECT. J Nucl Med 1994; 35:360-367.

51. Narita Y, Eberl S, Iida H, et al. Monte Carlo and experimental evaluation of accuracy and noise properties of two scatter correction methods for SPECT. Phys Med Biol 1996; 41:24812496.

52. Narita Y, Iida H, Eberl S, Nakamura T. Monte Carlo evaluation of accuracy and noise properties of two scatter correction methods for ${ }^{201} \mathrm{Tl}$ cardiac SPECT. IEEE Trans Nucl Sci 1997; 44:2465-2472.

53. Kim KM, Varrone A, Watabe H, et al. Contribution of scatter and attenuation compensation to SPECT images of nonuniformly distributed brain activities. J Nucl Med 2003; 44:512-519.

54. Larsson A, Johansson L. Scatter-to-primary based scatter fractions for transmission-dependent convolution subtraction of SPECT images. Phys Med Biol 2003; 48:N323-N328.
55. King MA, Hademenos GJ, Glick SJ. A dual-photopeak window method for scatter correction. J Nucl Med 1992; 33:605-612.

56. Ogawa K, Harata Y, Ichihara T, Kubo A, Hashimoto S. A practical method for position-dependent Compton-scatter correction in single-photon emission CT. IEEE Trans Med Imaging 1991; 10:408-412.

57. Koral KF, Wang XQ, Rogers WL, Clinthorne NH, Wang XH. SPECT Compton-scattering correction by analysis of energy spectra. J Nucl Med 1988; 29:195-202.

58. Wang X, Koral KF. A regularized deconvolution-fitting method for Compton-scatter correction in SPECT. IEEE Trans Med Imaging 1992; 11:351-360.

59. El Fakhri G, Kijewski MF, Maksud P, Moore SC. The effects of compensation for scatter, lead x-rays, and high-energy contamination on tumor detectability and activity estimation in Ga-67 imaging. IEEE Trans Nucl Sci 2003; 50:439-445.

60. Ogawa K, Nishizaki N. Accurate scatter compensation using neural networks in radionuclide imaging. IEEE Trans Nucl Sci 1993; 40:1020-1025.

61. Maksud P, Fertil B, Rica C, El Fakhri G, Aurengo A. Artificial neural network as a tool to compensate for scatter and attenuation in radionuclide imaging. J Nucl Med 1998; 39:735-745.

62. El Fakhri G, Moore SC, Maksud P. A new scatter compensation method for Ga-67 Imaging using artificial neural networks. IEEE Trans Nucl Sci 2001; 48:799-804.

63. Hutton B, Nuyts J, Zaidi H. Iterative image reconstruction methods. In: Zaidi $\mathrm{H}$, ed. Quantitative analysis in nuclear medicine imaging. New York: Kluwer Academic/Plenum Publishers, 2004.

64. Hutton BF, Baccarne V. Efficient scatter modelling for incorporation in maximum likelihood reconstruction. Eur $\mathrm{J}$ Nucl Med 1998; 25:1658-1665.

65. Kamphuis C, Beekman FJ, van Rijk PP, Viergever MA. Dual matrix ordered subsets reconstruction for accelerated 3D scatter compensation in single-photon emission tomography. Eur J Nucl Med 1998; 25:8-18.

66. Narayanan MV, Pretorius PH, Dahlberg ST, et al. Evaluation of scatter compensation strategies and their impact on human detection performance Tc-99m myocardial perfusion imaging. IEEE Trans Nucl Sci 2003; 50:1522-1527.

67. Koral KF, Dewaraja Y, Li J, et al. Update on hybrid conjugateview SPECT tumor dosimetry and response in 131I-tositumomab therapy of previously untreated lymphoma patients. J Nucl Med 2003; 44:457-464.

68. Iida H, Narita Y, Kado H, et al. Effects of scatter and attenuation correction on quantitative assessment of regional cerebral blood flow with SPECT. J Nucl Med 1998; 39:181-189.

69. Links JM. Scattered photons as "good counts gone bad:" are they reformable or should they be permanently removed from society? J Nucl Med 1995; 36:130-132.

70. Msaki P, Bentourkia M, Lecomte R. Scatter degradation and correction models for high-resolution PET. J Nucl Med 1996; 37:2047-2049.

71. Bailey DL. Quantitative procedures in 3D PET. In: Bendriem B, Townsend DW, eds. The theory and practice of 3D PET. Dordrecht, The Netherlands: Kluwer Academic; 1998:55-109.

72. Meikle SR, Badawi RD. Quantitative techniques in positron emission tomography. In: Valk PE, Bailey DL, Townsend DW, et al., eds. Positron emission tomography: basic science and clinical practice. London: Springer; 2003:115-146.

73. Grootoonk S, Spinks TJ, Sashin D, Spyrou NM, Jones T. Correction for scatter in 3D brain PET using a dual energy window method. Phys Med Biol 1996; 41:2757-2774. 
74. Bendriem B, Trebossen R, Frouin V, Syrota A. A PET scatter correction using simultaneous acquisitions with low and high lower energy thresholds. Proc IEEE Med Imag Conf, San Francisco, CA, 3:1779-1783.

75. Shao L, Freifelder R, Karp JS. Triple energy window scatter correction technique in PET. IEEE Trans Med Imaging 1994; 4:641-648.

76. Bentourkia M, Msaki P, Cadorette J, Lecomte R. Nonstationary scatter subtraction-restoration in high-resolution PET. J Nucl Med 1996; 37:2040-2046.

77. Bailey DL, Meikle SR. A convolution-subtraction scatter correction method for 3D PET. Phys Med Biol 1994; 39:411424.

78. Lercher MJ, Wienhard K. Scatter correction in 3D PET. IEEE Trans Med Imaging 1994; 13:649-657.

79. Cherry S, Huang SC. Effects of scatter on model parameter estimates in 3D PET studies of the human brain. IEEE Trans Nucl Sci 1995; 42:1174-1179.

80. Links JL, Leal JP, Mueller-Gartner HW, Wagner HN. Improved positron emission tomography quantification by Fourierbased restoration filtering. Eur J Nucl Med 1992; 19:925-932.

81. Ollinger JM. Model-based scatter correction for fully 3D PET. Phys Med Biol 1996; 41:153-176.

82. Wollenweber SD. Parameterization of a model-based 3-D PET scatter correction. IEEE Trans Nucl Sci 2002; 49:722-727.

83. Watson CC. New, faster, image-based scatter correction for 3D PET. IEEE Trans Nucl Sci 2000; 47:1587-1594.

84. Zaidi H, Montandon M-L, Slosman DO. Magnetic resonance imaging-guided attenuation and scatter corrections in threedimensional brain positron emission tomography. Med Phys 2003; 30:937-948.

85. Ferreira NC, Trebossen R, Lartizien C, Brulon V, Merceron P, Bendriem B. A hybrid scatter correction for 3D PET based on an estimation of the distribution of unscattered coincidences: implementation on the ECAT EXACT HR+. Phys Med Biol 2002; 47:1555-1571.

86. Zaidi H. Comparative evaluation of scatter correction techniques in 3D positron emission tomography. Eur J Nucl Med 2000; 27:1813-1826.

87. Daube-Witherspoon ME, Carson RE, Yan YC, Yap TK. Scatter correction in maximum-likelihood reconstruction of PET data. IEEE Nuclear Science Symposium and Medical Imaging Conference, 1992. Conference Record 2:945-947.

88. Werling A, Bublitz O, Doll J, Adam LE, Brix G. Fast implementation of the single scatter simulation algorithm and its use in iterative image reconstruction of PET data. Phys Med Biol 2002; 47:2947-2960.

89. Pan TS, Yagle AE. Numerical study of multigrid implementations of some iterative image reconstruction algorithms. IEEE Trans Med Imaging 1991; 10:572-588.

90. Ljungberg M, King MA, Hademenos GJ, Strand SE. Comparison of four scatter correction methods using Monte Carlo simulated source distributions. J Nucl Med 1994; 35:143151.

91. Buvat I, Rodriguez-Villafuerte M, Todd-Pokropek A, Benali H, Di Paola R. Comparative assessment of nine scatter correction methods based on spectral analysis using Monte Carlo simulations. J Nucl Med 1995; 36:1476-1488.
92. El Fakhri G, Buvat I, Benali H, Todd-Pokropek A, Di Paola R. Relative impact of scatter, collimator response, attenuation, and finite spatial resolution corrections in cardiac SPECT. J Nucl Med 2000; 41:1400-1408.

93. Townsend D, Price J, Mintun M, et al. Scatter correction for brain receptor quantitation in 3D PET. In: Myers R CVJ, Bailey DL, Jones T, eds. Quantification of brain function using PET. San Diego, CA: Academic Press; 1996:76-81.

94. Sossi V, Oakes TR, Ruth TJ. A phantom study evaluating the quantitative aspect of 3D PET imaging of the brain. Phys Med Biol 1998; 43:2615-2630.

95. Barrett HH, Denny JL, Wagner RF, Myers KJ. Objective assessment of image quality. II. Fisher information, Fourier crosstalk, and figures of merit for task performance. J Opt Soc Am A 1995; 12:834-852.

96. King MA, deVries DJ, Pan T-S, Pretorius PH, Case JA. An investigation of the filtering of TEW scatter estimates used to compensate for scatter with ordered subset reconstructions. IEEE Trans Nucl Sci 1997; 44:1140-1145.

97. Ljungberg M, Strand S-E, King MA. Monte Carlo calculations in nuclear medicine: applications in diagnostic imaging. Bristol: Institute of Physics Publishing, 1998.

98. Zaidi H, Sgouros G. Therapeutic applications of Monte Carlo calculations in nuclear medicine. Bristol: Institute of Physics Publishing, 2002.

99. Zaidi H. Relevance of accurate Monte Carlo modeling in nuclear medical imaging. Med Phys 1999; 26:574-608.

100. Kim KM, Watabe H, Shidahara M, Onishi Y, Yonekura Y, Iida $\mathrm{H}$. Impact of scatter correction in the kinetic analysis of a D2 receptror ligand SPECT study. IEEE Medical Imaging Conference, San Diego; 3:1509-1512.

101. Fujita M, Varrone A, Kim KM, et al. Measurement of striatal and extrastriatal dopamine $\mathrm{D}_{2}$ receptors using [ $\left.{ }^{123} \mathrm{I}\right]$ epidepride SPET. Eur J Nucl Med Mol Imaging 2004; 31: in press. DOI 10.1007/s00259-003-1431-7.

102. de Vries DJ, King MA, Soares EJ, Tsui BM, Metz CE. Effects of scatter subtraction on detection and quantitation in hepatic SPECT. J Nucl Med 1999; 40:1011-1023.

103. Sankaran S, Frey EC, Gilland KL, Tsui BM. Optimum compensation method and filter cutoff frequency in myocardial SPECT: a human observer study. J Nucl Med 2002; 43:432-438.

104. Montandon M-L, Slosman DO, Zaidi H. Assessment of the impact of model-based scatter correction on ${ }^{18} \mathrm{~F}-[\mathrm{FDG}] 3 \mathrm{D}$ brain PET in healthy subjects using statistical parametric mapping. Neuroimage 2003; 20:1848-1856.

105. Zaidi H, Hasegawa BH. Determination of the attenuation map in emission tomography. J Nucl Med 2003; 44:291-315.

106. Wegmann K, Adam L-E, Livieratos L, Zaers J, Bailey DL, Brix G. Investigation of the scatter contribution in single photon transmission measurements by means of Monte Carlo simulations. IEEE Trans Nucl Sci 1999; 46:1184-1190.

107. Celler A, Axen D, Togane D, El-Khatib J. Investigation of scatter in SPECT transmission studies. IEEE Trans Nucl Sci 2000; 47:1251-1257.

108. Ohnesorge B, Flohr T, Klingenbeck-Regn K. Efficient object scatter correction algorithm for third and fourth generation CT scanners. Eur Radiol 1999; 9:563-569. 